\title{
REFINED GRADIENT BOUNDS, POISSON EQUATIONS AND SOME APPLICATIONS TO OPEN KÄHLER MANIFOLDS *
}

\author{
BUN WONG ${ }^{\dagger}$ AND QI S. ZHANG ${ }^{\dagger}$
}

\begin{abstract}
Under some natural curvature assumptions on noncompact manifolds, we prove that the Poisson and Poincaré-Lelong equation $\Delta u=f$ and $\sqrt{-1} \partial \bar{\partial} u=\rho$ can be solved when $f$ and $\rho$ are in the long range. i.e. when they decay at a slower rate than $1 / d(x)$ near infinity. This extends, to the long range case, earlier results in [MSY] and [NST] which treated the case when $f$ decays faster than $1 / d(x)$. The improvement is based on a refined gradient estimate for harmonic and caloric functions. Some applications to the problems of curvature characterization of Stein manifolds are given.
\end{abstract}

1. Introduction. One of the most basic and important equations ever studied is the Poisson equation

$$
\Delta u=f
$$

in noncompact manifolds including $\mathbf{R}^{n}$. Here $\Delta$ is the Laplace-Beltrami operator. In this paper we study the problem of when (1.1) has a global classical solution. At the first glance, this problem seems well understood already. From any standard PDE book, one can find that (1.1) in $\mathbf{R}^{n}, n \geq 3$, always has a global solution if $|f(x)| \leq C /\left(1+|x|^{2+\delta}\right)$ with $\delta>0$. Moreover the solution can be written as $u(x)=$ $-\int_{\mathbf{R}^{n}} \Gamma(x, y) f(y) d y$. Here $\Gamma$ is the Green's function of the Laplacian in $\mathbf{R}^{n}$.

However, one can do a little better by considering

$$
u(x)=\int_{\mathbf{R}^{n}}[\Gamma(0, y)-\Gamma(x, y)] f(y) d y .
$$

It can be shown easily that the above is a solution of (1.1) provided that $|f(x)| \leq$ $C /\left(1+|x|^{1+\delta}\right)$ with $\delta>0$. This extends the range of $f$ by an order one. In fact, one can just set up an integral condition for $f$ so that (1.1) is solvable. This method was applied and generalized in the important papers by Mok, Siu, Yau [MSY] and Ni, Shi, Tam [NST] to the case of noncompact Riemannian manifolds with nonnegative Ricci curvatures. They used a version of formula (1.2) to derive interesting existence results on (1.1) and the related Poincaré-Lelong equation

$$
\sqrt{-1} \partial \bar{\partial} u=\rho .
$$

Roughly speaking, the decay condition for $f$ is $|f(x)| \leq \frac{C}{1+d(x)^{1+\delta}}$ in [MSY], and in [NST], its integral form $\int_{0}^{\infty} \frac{1}{|B(x, r)|} \int_{B(x, r)}|f| d y d r<\infty$. Here $d(x)$ is the distance from $x$ to a point on the manifold. $B(x, r)$ is the geodesic ball of radius $r$ centered at $x$ and $|B(x, r)|$ is the volume. The authors then apply their existence results to obtain several important geometric applications. These include, gap theorems, vanishing results and Steinness of manifolds.

The purpose of this paper is to show that, under some natural curvature assumptions, (1.1) and (1.3) can be solved even when $f$ decays slower than $\frac{C}{1+d(x)^{1-\epsilon}}, \epsilon>0$. In some special cases we can show that (1.1) can be solved even when $f$ blows up

\footnotetext{
*Received May 13, 2003; accepted for publication July 8, 2003.

†Department of Mathematics, University of California, Riverside, CA 92521, USA (wong@math.ucr.edu; qizhang@math.ucr.edu).
} 
near infinity completely. The current result differs from the previous ones in that we allow $f$ to be long range functions. Historically functions decaying faster than $c / d(x)$ near infinity are called short range potentials while those decaying slower than $C / d(x)$ are called long range ones. Even the border line case $f \sim C / d(x)$ is very interesting. In fact one of the motivations of the paper is to understand when can one solve the equation $\Delta u(x)=\frac{1}{1+d(x)}$ on a manifold. This kind of problems deserve some special attention since they appear naturally during a fundamental operation i.e. when one computes the Laplacian of the distance function $r$. Recall $\Delta r=\frac{n-1}{r}+\partial_{r} \ln \sqrt{g}$. Here $\sqrt{g}$ is the volume element of the manifold.

In order to solve (1.1) and (1.3) with long range data, some new information is needed. One needs additional decays for the derivative of the Green's functions. Let $G$ be the heat kernel of the Laplacian in M. Suppose the Ricci curvature is nonnegative. From the classical result by $\mathrm{Li}$ and Yau [LY] and others, one has

$$
\begin{gathered}
G(x, t ; y, 0) \leq \frac{C}{|B(x, \sqrt{t})|} e^{-d(x, y)^{2} /(4+c) t}, \\
\left|\nabla_{x} G(x, t ; y, 0)\right| \leq \frac{C}{\sqrt{t}|B(x, \sqrt{t})|} e^{-d(x, y)^{2} /(4+c) t} .
\end{gathered}
$$

These very useful bounds provide a major tool in geometric analysis and they are sharp in general, even in $\mathbf{R}^{n}$.

Nevertheless, we discover that the gradient bound can be refined if the Ricci curvature satisfies a natural lower bound: a multiple of the inverse square of the distance function $\frac{a}{1+d(x)^{2}}, a>0$. It is well known that this is a large class of manifolds (see Remark 1.3 below). A strong motivation for studying this kind of manifold comes from the well known results that roughly claims: a manifold whose sectional curvatures are nonnegative and decaying faster than the inverse square of the distance function is flat.

What we are studying is, in some sense, the complement of the above result. i.e. what are the differences between $\mathbf{M}$ and $\mathbf{R}^{n}$ if the curvature assumption in the above theorem fails. We find that, under the curvature assumption $\left(\operatorname{Ric}(x) \geq a\left(1+d(x)^{2}\right)>\right.$ 0 ), the analytic properties of the manifold are indeed quite different from those of $\mathbf{R}^{n}$. For example the gradient of harmonic functions has additional decay comparing with the Euclidean case. It is the additional decay of the gradient that allows us to solve (1.1) and (1.3) for long range data. We expect the refined decay estimates will have further applications.

Basic assumptions.

In this paragraph we lay out a number assumptions and notations to be used through out the paper. Unless otherwise stated, $\mathbf{M}$ is a $n(\geq 3)$ dimensional complete noncompact Riemannian manifold with nonnegative Ricci curvature. Some times we will use the term nonparabolic manifolds. This means the Laplace-Beltrami operator admits a positive Green's function. An example is $\mathbf{R}^{\mathbf{n}}, n \geq 3$. Otherwise we call the manifold parabolic. Ric $(x)$ denotes the Ricci curvature at $x$ and $\operatorname{Scal}(x)$ notes the scalar curvature at $x$.

0 will be a reference point on $\mathbf{M}$ and $d(x, y), d(x)$ will be the distance between $x, y$ and between $x, 0$ respectively. The notation $Q_{r}(x, t)$ will be used to denote the parabolic cube $B(x, r) \times\left(t-r^{2}, t\right)$. We will use $c, C, c_{1}, C_{1}, \ldots$, to denote generic positive constants. 
To ensure the smoothness of solutions to (1.1) and (1.3), we always assume that $f$ and $\rho$ are locally Hölder continuous.

The following theorems are the main results of the paper.

TheOREM 1.1. (Refined gradient bounds) Let $\mathbf{M}$ be a complete, noncompact, Riemannian manifold. Let $G$ be the heat kernel of $\Delta$ in $\mathbf{M}$. Suppose, for $a>0$, $\operatorname{Ric}(x) \geq \frac{a}{1+d^{2}(x)}$. Then, given any $c>0$, there exist $C>0$ such that, for $\alpha=\alpha(a)=$ $\left[64(n+4)^{2}\right]^{-1} a$ and all $x, y, t>0$,

$$
\left|\nabla_{x} G(x, t ; y, 0)\right| \leq C\left(\frac{1+\sqrt{t}}{1+d(x)} \vee 1\right)^{-\alpha} \frac{1}{\sqrt{t}|B(x, \sqrt{t})|} \exp \left(-\frac{d(x, y)^{2}}{(4+c) t}\right)
$$

REMARK 1.1. In general Theorem 1.1 does not hold if the Ricci curvature does not satisfy the stated lower bound. For example it does not hold in $\mathbf{R}^{n}$. We need to point out that the constant $a$ can not be too large. By a generalization of BonnetMyers theorem, if $\operatorname{Ric}(x) \geq \frac{a}{1+d(x)^{2}}$ and $a>1 / 4$, then $\mathbf{M}$ is compact. This fact is implied by the main theorem in [C]. Therefore $\alpha(a)$ is smaller than 1 in Theorem 1.1 here. This constant can be better estimated if more information on the manifold is available. This includes, for example, the growth rate of geodesic balls at infinity. However the linear dependence of $\alpha$ on $a$ is qualitatively sharp. This point will be clear from the proof. An example of a manifold satisfying the assumption of the theorem is given in Remark 1.3.

TheOREM 1.2. Suppose $\mathbf{M}$ is a noncompact manifold such that, for some $a>0$, $\operatorname{Ric}(x) \geq \frac{a}{1+d(x)^{2}}$. Then the following conclusions hold.

(a). For the same $\alpha$ as in Theorem 1.1, the Poisson equation $\Delta u=f$ has a solution in $\mathbf{M}$ provided that $\sup _{x \in \mathbf{M}} \int_{0}^{\infty} k(x, r) r^{-\alpha} d r<\infty$. Here and later

$$
k(x, r) \equiv \frac{1}{|B(x, r)|} \int_{B(x, r)}|f(y)| d y .
$$

In particular, this includes $f$ such that $|f(x)| \leq \frac{C}{1+d(x)^{1-\epsilon}}$ for any $\epsilon<\alpha$.

(b). $|u(x)| \leq C(1+d(x))^{1+\alpha}$.

(c). $|\nabla u(x)| \leq C(1+d(x))^{\alpha}$.

(d). $\lim _{R \rightarrow \infty}|B(0, R)|^{-1} \int_{B(0, R)}\left|\nabla^{2} u\right|^{2}=0$.

'THEOREM 1.2'. Let $\mathbf{M}$ be a noncompact Kähler manifold with nonnegative holomorphic bisectional curvature. Suppose, for some $a>0, \operatorname{Ric}(x) \geq \frac{a}{1+d(x)^{2}}$.

Let $\rho$ be a real closed $(1,1)$. Suppose $\rho$ satisfies

(a) $\sup _{x \in \mathrm{M}} \int_{0}^{\infty} \frac{r^{-\alpha}}{|B(x, r)|} \int_{B(x, r)}\|\rho\| d x d r<\infty$,

(b) $\lim _{r \rightarrow \infty} \frac{1}{|B(0, r)|} \int_{B(0, r)}\|\rho\|^{2} d x=0$.

Then exists a solution to the Poincaré-Lelong equation $\sqrt{-1} \partial \bar{\partial} u=\rho$ in $\mathbf{M}$.

REMARK 1.2. In particular, the conditions in Theorem 1.2' admit those $\rho$ satisfying $\|\rho(x)\| \leq \frac{C}{1+d(x)^{1-\epsilon}}$ for any $\epsilon<\alpha$. Note that for any $a>0$, Theorem 1.2 and 1.2 ' covers all $f$ or $\rho$ such that $|f(x)|,\|\rho(x)\| \leq \frac{C}{1+d(x)}$. In this sense, our theorems establish an end point type results for [MSY] and [NST], for a large class of manifolds. 
It is an open problem whether a complete open Kähler manifold with positive bisectional curvature is a Stein manifold. It was proved in Greene and Wu [GW1] that it is true when the sectional curvature is positive. Moreover the following result was first established by $\mathrm{Wu}$ as a special case of Theorem 4 [Wu2].

TheOREM (H. Wu). Let $\mathbf{M}$ be a complete open Kähler manifold of positive bisectional curvature with a pole. Then $\mathbf{M}$ is a Stein manifold.

The proof goes roughly as follows. $\mathbf{M}$ is simply since it has a pole. Let $p$ be the pole of $\mathbf{M}$. Then $\mathbf{M}$ is covered by all the rays $\gamma$ starting from $p$. Using this fact one can easily conclude (see [GW2]) the distance function $d(p, x)=\sup _{\gamma}\left\{g_{\gamma}(x)\right\}$ is a continuous strictly plurisubharmoic function, where $g_{\gamma}$ is the Buseman function defined with respect to $\gamma$. But $d(p, x)$ is an exhaustion function as $\mathbf{M}$ is complete. Invoking Narasimhan's continuous version of Grauert's criterion, one concludes $\mathbf{M}$ is Stein.

There are obvious counter examples to the above open question if one merely assumes $\mathbf{M}$ admits nonnegative bisectional curvature (or nonnegative sectional curvature). However combining [GW1] with Theorem 1.2', one immediately obtains

TheOREM 1.3. (a). Let $\mathbf{M}$ be a complete open Kähler manifold with nonnegative sectional curvature. Suppose, for $a>0, \frac{a}{1+d(x)^{2}} \leq \operatorname{Ric}(x)$ and $\operatorname{Scal}(x) \leq \frac{C}{1+d(x)^{1-\epsilon}}$ for $\epsilon<\alpha(a)=\left[64(n+4)^{2}\right]^{-1} a$. Then $\mathbf{M}$ is Stein.

The same holds if Ric(x) satisfies just the lower bound and there exists a real closed $(1,1)$ form $\rho$ which is positive everywhere and satisfying $(a)$ and $(b)$ in Theorem 1.2'.

(b). Let $\mathbf{M}$ be a complete open Kähler manifold with nonnegative bisectional curvature and with a pole. Suppose, for $a>0, \frac{a}{1+d(x)^{2}} \leq \operatorname{Ric}(x)$ and $\operatorname{Scal}(x) \leq$ $\frac{C}{1+d(x)^{1-\epsilon}}$ for $\epsilon<\alpha(a)=\left[64(n+4)^{2}\right]^{-1} a$. Then $\mathbf{M}$ is Stein.

Theorem 1.3 (b) provides a partial answer to a question raised in [Wu2], p255 "Is a Kähler manifold with a pole whose bisectional curvature is nonnegative necessarily a Stein manifold?".

It is worth comparing Theorem 1.3 here with the interesting Theorem 5.2 (ii) in [NST]. It is proved there that if $\mathbf{M}$ has nonnegative sectional curvature and there exists a real closed $(1,1)$ form $\rho$ which is positive everywhere and satisfying

1) $\sup _{x \in \mathrm{M}} \int_{0}^{\infty} \frac{1}{|B(x, r)|} \int_{B(x, r)}\|\rho\| d x d r<\infty$

2) $\lim _{r \rightarrow \infty} \frac{1}{|B(0, r)|} \int_{B(0, r)}\|\rho\|^{2} d x=0$,

then $\mathbf{M}$ is Stein. An example of such $\rho$ is the Ricci curvature provided it is positive and has fast decay. In contrast, we allow the Ricci curvature to have slow decay. However, we have a pay the price by assuming the Ricci curvature is bounded below by inverse square of the distance. A similar comparison to a recent paper [CZ] is also valid. According to [NST], it is proved there ([CZ]) that if $\mathbf{M}$ has nonnegative holomorphic bisectional curvature, has maximum volume growth such that the scalar curvature $R$ satisfies $R(x) \leq C d(x)^{-1-\epsilon}$ for some constants $C$ and $\epsilon>0$ for all $x$, then $\mathbf{M}$ is Stein. These results are motivated by the earlier works [MSY], $[\mathrm{M}]$ and [GW1], for which we refer the reader to [NST] for a nice discussion.

REMARK 1.3. There are many Kähler manifolds satisfying the conditions of Theorem 1.3. Here we give a simple example of a Kähler manifold of nonnegative sectional curvature, whose Ricci curvature is pinched between $\frac{a}{1+d(x)^{2}}$ and $\frac{C}{1+d(x)}$. 
Let $\mathbf{M}$ be $\mathbf{R}^{n}$ equipped with a rotationally symmetric metric tensor $g=d r^{2}+$ $\phi^{2}(r) d^{2} S_{n-1}$, where $n=2 m$ is even and $\phi=\int_{0}^{r} \frac{1}{\left(1+s^{2}\right)^{1 / 4}} d s$. It is easy to check, this defines a complete metric and $\phi(r) \sim \sqrt{r}$ when $r$ is large. Moreover $-\phi^{\prime \prime}(r) / \phi(r) \geq$ $\frac{a}{1+r^{2}}>0, \phi^{\prime}(r) \leq 1$ and $\frac{1-\left(\phi^{\prime}\right)^{2}}{\phi^{2}} \sim \frac{1}{1+r}$.

Choosing an orthonormal frame $\left\{E_{1}, \ldots, E_{n-1}\right\}$ on $S_{n-1}$, then

$$
\left\{F_{1}, \ldots, F_{n}\right\} \equiv\left\{\phi^{-1} E_{1}, \ldots, \phi^{-1} E_{n-1}, \partial_{r}\right\}
$$

is an orthonormal frame on $(\mathbf{M}, g)$. Following the standard formulas

$$
\begin{gathered}
\operatorname{Ric}\left(F_{i}\right)=\left((n-2) \frac{1-\left(\phi^{\prime}\right)^{2}}{\phi^{2}}-\frac{\phi^{\prime \prime}}{\phi}\right) F_{i}, i<n, \operatorname{Ric}\left(F_{n}\right)=-(n-1) \frac{\phi^{\prime \prime}}{\phi} F_{n}, \\
\text { scalar curvature }=-2(n-1) \frac{\phi^{\prime \prime}}{\phi}+(n-1)(n-2) \frac{1-\left(\phi^{\prime}\right)^{2}}{\phi^{2}}
\end{gathered}
$$

By the estimates on $\phi^{\prime}$ and $\phi^{\prime \prime}$, we see that $\frac{a}{1+d(x)^{2}} \leq \operatorname{Ric}(x)$ and $\operatorname{scal}(x) \sim \frac{C}{1+d(x)}$.

We are going to show that this $\mathbf{M}$ is a Hermitian manifold. Let $x=\left(x_{1}, . ., x_{n}\right)$ be the Euclidean coordinate of $\mathbf{R}^{n}$. Write $s=\sqrt{x_{1}^{2}+\ldots+x_{n}^{2}}$. Define a function $f=f(s)$ by the relations $\phi(r)=s f(s)$ and $r=\int_{0}^{s} f(\tau) d \tau, f(0)=1, f^{\prime}(0)=0$. Here $\phi$ is given in the last paragraph. This is equivalent to solving the differential equation $r^{\prime}(s)=\phi(r) / s$. Since $\phi$ is monotone, $\phi(0)=0$ and $\phi^{\prime}(0)=1$, by direct computation we know that $f$ is a smooth function such that $f(s) \geq c /(1+s)>0$. It is easy to see that $g=f^{2}\left(\sqrt{x_{1}^{2}+\ldots+x_{n}^{2}}\right)\left(d^{2} x_{1}+\ldots+d^{2} x_{n}\right)$. Hence $g$ is Hermitian. It is well known that such $\mathbf{M}$ with $n=2$ is a complete Kähler manifold. The direct product of this manifold with itself is also a Kähler manifold satisfying all assumptions in Theorem 1.3 .

Using similar arguments, we also have

OBSERVATION. Let $\mathbf{M}$ be a complete open Kähler manifold with nonnegative bisectional curvature and maximum volume growth i.e. $|B(x, r)| \geq c r^{2 n}$ for some $c>0$ and all $x$ and $r>0$. Here $n$ is the complex dimension of $\mathbf{M}$. Then the following results hold

(a) Suppose the bisectional curvature is positive outside a compact set. Then $\mathbf{M}$ is a Stein.

(b) Suppose, for $a>0, \frac{a}{1+d(x)^{2}} \leq \operatorname{Ric}(x)$ and Scal $(x) \leq \frac{C}{1+d(x)^{1-\epsilon}}$ for $\epsilon<\alpha(a)=$ $\left[64(n+4)^{2}\right]^{-1} a$. Then $\mathbf{M}$ is Stein.

Here is the proof.

(a) The Ricci curvature satisfies the conditions in Shen's theorem [S] because the bisectional curvature is so assumed. i.e. The Ricci curvature is nonnegative and positive outside a compact set. The spherical Busemann function $b_{p}(x)$, defined as

$$
\lim _{r \rightarrow \infty}\{r-d(x, \partial B(p, r)\}
$$

is a continuous plurisubharmonic exhaustion by Shen's theorem and a result due to $\mathrm{H}$. Wu ([Wu1]). Here $p$ is a fixed point in $\mathbf{M}$. It also follows from the same paper (Theorem $\mathrm{C}[\mathrm{Wu} 1]$ ) that there exists a strictly plurisubharmonic function in $\mathbf{M}$. This finishes the proof. 
(b) In this case, the existence of a strictly plurisubharmonic function follows from Theorem 1.2' as to be show in the proof of Theorem 1.3 (b). Consequently M is Stein for the same reason as above.

Note that there is no assumption that $\mathbf{M}$ has a pole in the above observation . However we need the assumption of maximum growth. Part (a) is basically a direct consequence of a remarkable result due to Z. M. Shen [S]. There are a lot of examples satisfying condition (a) in the observation. However we are not able to construct a manifold satisfying (b). (The anonymous referee informs us that it may not exist.)

The rest of the paper is organized as follows. In section 2 we will prove the refined gradient bounds for harmonic functions (Theorem 1.1.). In section 3 we will prove Theorem 1.2 and 1.2' concerning the solvability of (1.1) and (1.3). Applications (Theorems 1.3) will also be proven in this section. In section 4 we will present a generalization of the theorems to some flat manifolds.

2. Refined gradient bounds. In this section we prove Theorem 1.1 and establish an upper bound on the integral of the gradient of the heat kernel.

The proof, which is somewhat.involved, requires a number of preliminary results to be listed as propositions and lemmas below. Here is an outline. Let $G$ be the heat kernel of the Laplacian in $\mathbf{M}$ and let $u(x, t) \equiv G(x, t ; y, 0)$. By Bochner technique, it is known that $w=|\nabla u|$ is a sub-solution to a Schrödinger heat equation i.e. $\Delta w-$ $V w-w_{t} \geq 0$. Here $V=V(x)$ is the lower bound of the Ricci curvature. The idea is to exploit decay property of $V$ to deduce additional decays for $w$. We remark that this theorem might be proved by using a weighted $L^{2}$ space method. But that method seems to require more assumptions on the manifold, to say the least.

Through out the paper we will frequently use the following well-known inequalities which are simple consequences of the doubling properties of geodesic balls.

$$
\frac{e^{-c d(x, y)^{2} / t}}{\sqrt{\left|B\left(x, t^{1 / 2}\right)\right|} \sqrt{\left|B\left(y, t^{1 / 2}\right)\right|}} \leq C \frac{e^{-c d(x, y)^{2} /(2 t)}}{\left|B\left(x, t^{1 / 2}\right)\right|} \leq C \frac{e^{-c d(x, y)^{2} /(4 t)}}{|B(x, d(x, y))|}
$$

Proposition 2.1. Let $\mathbf{M}$ be a complete, noncompact manifold with nonnegative Ricci curvature. Let $G_{V}$ be the fundamental solution to the equation $\Delta u-V u-u_{t}=0$ in $\mathbf{M} \times(\mathbf{0}, \infty)$.

Suppose, for a $>0, V(x) \geq \frac{a}{1+d^{2}(x)}$. Then there exist positive constants $c_{1}, c_{2}$ such that, for all $x, y$ and $t>0$,

$$
G_{V}(x, t ; y, 0) \leq c_{1} \frac{w(x, t)}{\sqrt{\left|B\left(x, t^{1 / 2}\right)\right|}} \frac{w(y, t)}{\sqrt{\left|B\left(y, t^{1 / 2}\right)\right|}} e^{-c_{2} d(x, y)^{2} / t} .
$$

Here $w(x, t)=\left[\max \left\{\frac{t^{1 / 2}}{1+d(x)}, 1\right\}\right]^{-\alpha}$ with $\alpha=\alpha(a)=\min \left\{\left[64(n+4)^{2}\right]^{-1} a, 1\right\}$.

Part of this result (assuming $a$ is sufficiently large) was proven in [Zh]. Now we are able to prove it for any $a>0$. The proof of the upper bound is based on an explicit estimate on the $L^{\infty}$ bounds of solutions to the parabolic equation. The key is to find a precise relation between the bounds and the potential. This requires us to refine Moser's iteration scheme to capture the information on the potential.

Let $u$ be a solution of $\Delta u-V u-u_{t}=0$. If $V \geq 0$, by Moser's iteration and the maximal principle, we have, for $C>0$,

$$
u^{2}(x, t) \leq \frac{C}{\left|Q_{r}(x, t)\right|} \int_{Q_{r}(x, t)} u^{2}(y, s) d y d s
$$


where $Q_{r}(x, t)$ is a parabolic cube defined in the introduction. However the next lemma shows that the constant $C$ can be refined if one knows the decay property of $V$.

Before proving the proposition, we need

Lemma 2.1. (crude mean value formula) Let $u$ be a solution to the equation $\Delta u-V u-u_{t}=0$ in $Q_{2 r}(x, t) \subset \mathbf{M} \times(0, \infty)$. Here $\mathbf{M}$ is as in Proposition 2.1.

Let $a \geq a_{0} \equiv 64(n+4)^{2}$. Suppose

$$
V(x) \geq \frac{a}{1+d^{2}(x)} .
$$

Then for positive $C$ depending on a but independent of $r$,

$$
u^{2}(x, t) \leq \frac{C[\max \{r /(1+d(x)), 1\}]^{-2}}{\left|Q_{r}(x, t)\right|} \int_{Q_{r}(x, t)} u^{2}(y, s) d y d s .
$$

Proof. We will use a trick in [Zh]. Given $\tau>1$, we pick a Lipschitz cut-off function $\phi$ such that $\phi(y, s)=1$ when $(y, s) \in Q_{r}(x, t), \phi(y, s)=0$ when $(y, s) \in Q_{i r}^{c}(x, t)$, and $|\nabla \phi| \leq 1 /((\tau-1) r)$, a.e. $\left|\partial_{t} \phi\right| \leq 1 /((\tau-1) r)^{2}$, a.e. Using $\phi^{2} u$ as a test function we obtain, after routine calculation,

$$
\int|\nabla(\phi u)|^{2} d y d s+\int V(y) u^{2} \phi^{2} d y d s \leq \int u^{2}\left[|\nabla \phi|^{2}+\left|\partial_{s} \phi\right|\right] d y d s .
$$

Therefore

$$
\int_{Q_{\tau r}(x, t)} V(y) u^{2} \phi^{2} d y d s \leq \frac{1}{((\tau-1) r)^{2}} \int_{Q_{\tau r}(x, t)} u^{2} d y d s
$$

When $y \in B(x, r)$ we have $d(y)^{2} \leq 2\left(d(x, y)^{2}+d(x)^{2}\right)$ and hence

$$
V(y) \geq \frac{a}{2\left(d(x, y)^{2}+d(x)^{2}\right)} \geq \frac{a}{2\left(r^{2}+d(x)^{2}\right)} .
$$

It follows that

$$
\int_{Q_{r}(x, t)} u^{2} d y d s \leq \frac{2 a^{-1}\left(r^{2}+d(x)^{2}\right)}{(\tau-1)^{2} r^{2}} \int_{Q_{\tau r}(x, t)} u^{2} d y d s .
$$

For each $r>1$ we take $\tau>1$ such that

$$
\frac{2 a^{-1}\left(r^{2}+d(x)^{2}\right)}{(\tau-1)^{2} r^{2}}=\frac{1}{2} .
$$

This implies

$$
\tau r=r+\left[4 a^{-1}\left(r^{2}+d(x)^{2}\right)\right]^{1 / 2} .
$$

Under such a choice of $\tau$, we have

$$
\int_{Q_{r}(x, t)} u^{2} d y d s \leq \frac{1}{2} \int_{Q_{\tau r}(x, t)} u^{2} d y d s .
$$

We shall iterate the above inequality according to the formula

$$
r_{k+1}=\tau_{k} r_{k} \equiv r_{k}+\left[4 a^{-1}\left(r_{k}^{2}+d(x)^{2}\right)\right]^{1 / 2}
$$


with $r_{0}=d(x)$. Writing $\mu \equiv \sqrt{4 a^{-1}}$, we claim that there exist positive constant $C$ such that

$$
r_{k} \leq(1+\mu)^{2 k}(1+d(x)) .
$$

Obviously (2.5) hold for $k=1$. Suppose it holds for $k$, then

$$
\begin{aligned}
r_{k+1} & \leq r_{k}+\mu\left(r_{k}+d(x)\right)=(1+\mu) r_{k}+\mu d(x) \\
& \leq(1+\mu)^{2 k+1}(1+d(x))+(1+\mu)^{2 k+1} \mu(1+d(x)) \\
& \leq(1+\mu)^{2 k+2}(1+d(x)) .
\end{aligned}
$$

This implies that to reach $r$ from $d(x)$ one needs at least

$$
k=\frac{\ln (r /(1+d(x))}{2 \ln (1+\mu)}
$$

number of iterations (round up to an integer). Iterating (2.4) $k$ times we have

$$
\begin{aligned}
u(x, t)^{2} & \leq \frac{C}{\left|Q_{r_{0}}(x, t)\right|} \int_{Q_{r_{0}}(x, t)} u^{2}(y, s) d y d s \leq \frac{C 2^{-k}}{\left|Q_{r_{0}}(x, t)\right|} \int_{Q_{r}(x, t)} u^{2}(y, s) d y d s \\
& \leq C e^{-\frac{\ln [r /(1+d(x))]}{\ln (1+\mu)} \frac{\ln 2}{2}} \frac{\left(r / r_{0}\right)^{n+2}}{\left|Q_{r}(x, t)\right|} \int_{Q_{r}(x, t)} u^{2}(y, s) d y d s
\end{aligned}
$$

Here we also have used the volume comparison theorem, i.e. $|B(x, r)| \leq$ $c\left(\frac{r}{r_{0}}\right)^{n}\left|B\left(x, r_{0}\right)\right|$. Simplifying the above, we reach

$$
u(x, t)^{2} \leq C\left(\frac{r}{1+d(x)}\right)^{-\ln 2 /[2 \ln (1+\mu)]}\left(\frac{r}{1+d(x)}\right)^{n+2} \frac{1}{\left|Q_{r}(x, t)\right|} \int_{Q_{r}(x, t)} u^{2}(y, s) d y d s
$$

Recall that $\mu=\sqrt{4 a^{-1}}$. When $a \geq 64(n+4)^{2}$, we have

$$
\mu \leq \sqrt{4 /\left(64(n+4)^{2}\right)}=1 / 4(n+4) .
$$

Hence

$$
\frac{\ln 2}{2 \ln (1+\mu)} \geq \frac{\ln 2}{2 \mu}=(n+4) 2 \ln 2 \geq n+4
$$

This shows .

$$
u(x, t)^{2} \leq C\left(\frac{r}{1+d(x)}\right)^{-2} \frac{1}{\left|Q_{r}(x, t)\right|} \int_{Q_{r}(x, t)} u^{2}(y, s) d y d s
$$

This proves the lemma.

Note the above estimate is useful only when $\frac{r}{1+d(x)} \geq 1$ since standard estimate is already better otherwise.

Later in the section we are going to remove the largeness assumption on $a$.

Using the above mean value property, we are going to prove the global bounds for the Schrödinger heat kernel $G_{V}$.

Proof of Proposition 2.1. We divide the proof into two cases. 
Case 1. $V(x) \geq a /\left(1+d(x)^{2}\right)$ with $a \geq a_{0}=64(n+4)^{2}$.

The proof of the upper bound in this case is reduced to the standard method. For this reason we will be brief. For a fixed $\lambda \in \mathbf{R}$ and a fixed bounded function $\psi$ such that $|\nabla \psi| \leq 1$, we write

$$
f_{s}(y)=e^{\lambda \psi(y)} \int G_{V}(y, s ; z, 0) e^{-\lambda \psi(z)} f(z) d z .
$$

Direct computation shows that

$$
\partial_{s}\left\|f_{s}\right\|_{2}^{2} \leq-C\left\|\nabla f_{s}\right\|_{2}^{2}+C \lambda^{2}\left\|f_{s}\right\|_{2}^{2}-\int V(y) f_{s}^{2} d y .
$$

Since $V \geq 0$ we have

$$
\partial_{s}\left\|f_{s}\right\|_{2}^{2} \leq C \lambda^{2}\left\|f_{s}\right\|_{2}^{2}
$$

which implies

$$
\left\|f_{s}\right\|_{2}^{2} \leq e^{C \lambda^{2} s}\|f\|_{2}^{2}
$$

Now consider the function

$$
u(y, s)=e^{-\lambda \psi(y)} f_{s}(y)
$$

which is a solution to $\Delta u-V u-\partial_{t} u=0$ in $\mathbf{M} \times(\mathbf{0}, \infty)$. Applying Lemma 2.1 with $Q_{t^{1 / 2} / 2}(x, t)=B_{t^{1 / 2} / 2}(x) \times(3 t / 4, t)$, we obtain

$$
u(x, t)^{2} \leq C \frac{w_{2}(x, t)}{\left|Q_{t^{1 / 2} / 2}(x, t)\right|} \int_{3 t / 4}^{t} \int_{B\left(x, t^{1 / 2} / 2\right)} u^{2},
$$

where

$$
w_{2}(x, t) \equiv[\max \{\sqrt{t} /(1+d(x)), 1\}]^{-2} .
$$

It follows that

$$
\begin{aligned}
e^{2 \lambda \psi(x)} u(x, t)^{2} & \leq C e^{2 \lambda \psi(x)} \frac{w_{2}(x, t)}{\left|Q_{t^{1 / 2} / 2}(x, t)\right|} \int_{3 t / 4}^{t} \int_{B\left(x, t^{1 / 2} / 2\right)} u^{2} \\
& =C \frac{w_{2}(x, t)}{\left|Q_{t^{1 / 2} / 2}(x, t)\right|} \int_{3 t / 4}^{t} \int_{B\left(x, t^{1 / 2} / 2\right)} e^{2 \lambda[\psi(x)-\psi(z)]} f_{s}^{2} \\
& \leq C e^{2 \lambda t^{1 / 2}} \frac{w_{2}(x, t)}{\left|B\left(x, t^{1 / 2}\right)\right|} e^{C \lambda^{2} t}\|f\|_{2}^{2}
\end{aligned}
$$

Taking the supremum over all $f \in L^{2}\left(B\left(y, t^{1 / 2}\right)\right)$ with $\|f\|_{2}=1$, we find that

$$
e^{2 \lambda[\psi(x)-\psi(y)]} \int_{B\left(y, t^{1 / 2} / 2\right)} G_{V}(x, t ; z, 0)^{2} d z \leq C e^{4 \lambda t^{1 / 2}+C \lambda^{2} t} \frac{w_{2}(x, t)}{\left|B\left(x, t^{1 / 2}\right)\right|} .
$$

Using Lemma 2.1 on the second entries of the heat kernel we have

$$
\begin{aligned}
G_{V}(x, t ; y, 0)^{2} & \leq C \frac{w_{2}(y, t)}{\left|Q_{t^{1 / 2} / 2}(y, t)\right|} \int_{0}^{t / 4} \int_{B\left(y, t^{1 / 2} / 2\right)} G_{V}(x, t ; z, s)^{2} d z d s \\
& \leq C \frac{w_{2}(x, t)}{\left|B\left(x, t^{1 / 2}\right)\right|} \frac{w_{2}(y, t)}{\left|B\left(y, t^{1 / 2}\right)\right|} e^{4 \lambda t^{1 / 2}+C \lambda^{2} t-2 \lambda[\psi(x)-\psi(y)]}
\end{aligned}
$$


Choosing $\lambda=d(x, y) / C t$ and $\psi$ such that $\psi(x)-\psi(y)=d(x, y)$, we reach

$$
G(x, t ; y, 0)^{2} \leq C \frac{w_{2}(x, t)}{\left|B\left(x, t^{1 / 2}\right)\right|} \frac{w_{2}(y, t)}{\left|B\left(y, t^{1 / 2}\right)\right|} e^{-c d(x, y)^{2} / t} .
$$

This is the desired bound.

Case 2. $V(x) \geq a /\left(1+d(x)^{2}\right)$ with $a>0$ being arbitrary.

Given $\lambda>1$, let $G_{1}$ and $G$ be the heat kernel of $\Delta-\lambda V$ and $\Delta$ respectively. By the Feynman-Kac or the Trotter product formula, we have

$$
G_{V}(x, t ; y, 0) \leq\left[G_{1}(x, t ; y, 0)\right]^{1 / \lambda}[G(x, t ; y, 0)]^{1-(1 / \lambda)} .
$$

For completeness we provide a sketch of the proof. For a given $f \in C_{0}^{\infty}(\mathbf{M})$,

$$
\begin{aligned}
& \int_{\mathbf{M}} G_{V}(x, t ; y, 0) f(y) d y=e^{(\Delta-V) t} f \\
& =\lim _{k \rightarrow \infty}\left[e^{\Delta \frac{t}{k}} e^{-V \frac{t}{k}}\right]^{k} f \\
& =\lim _{k \rightarrow \infty} \int_{\mathbf{M}} \ldots \int_{\mathbf{M}} G\left(x, \frac{t}{k} ; x_{k-1}, 0\right) \ldots G\left(x_{1}, \frac{t}{k} ; x_{0}, 0\right) e^{-\frac{t}{k} \Sigma_{0}^{k-1} V\left(x_{j}\right)} f\left(x_{0}\right) d x_{k-1} \ldots d x_{0} \\
& =\lim _{k \rightarrow \infty} \int_{\mathbf{M}} \ldots \int_{\mathbf{M}} G^{(1 / \lambda)} \ldots G^{(1 / \lambda)} e^{-\frac{t}{k} \Sigma_{0}^{k-1} V\left(x_{j}\right)} f^{(1 / \lambda)}\left(x_{0}\right) \\
& \leq \lim _{k \rightarrow \infty}\left[\int_{\mathbf{M}} \ldots \int_{\mathbf{M}} G \ldots G e^{-\lambda \frac{t}{k} \Sigma_{0}^{k-1} V\left(x_{j}\right)} f\left(x_{0}\right) d x_{k-1} \ldots d x_{0}\right]^{(1 / \lambda)} \\
& \quad \times\left[\int_{\mathbf{M}} \ldots \int_{\mathbf{M}} G \ldots G f\left(x_{0}\right) d x_{k-1} \ldots d x_{0}\right]^{1-(1 / \lambda)} \ldots G^{1-(1 / \lambda)} f^{1-(1 / \lambda)}\left(x_{0}\right) d x_{k-1} \ldots d x_{0} \\
& =\left[\int_{\mathbf{M}} G_{1}(x, t ; y, 0) f(y) d y\right]^{(1 / \lambda)}\left[\int_{\mathbf{M}} G(x, t ; y, 0) f(y) d y\right]^{1-(1 / \lambda)} .
\end{aligned}
$$

In the above we have used Hölder's inequality. The desired inequality is obtained by taking $f$ to be a Dirac delta function.

Taking $\lambda=a_{0} / a$, then $\lambda V(x) \geq \frac{a_{0}}{1+d(x)^{2}}$. Hence we can apply the result in case 1 to $G_{1}$ to reach

$$
G_{1}(x, t ; y, 0) \leq C \frac{(\sqrt{t} /(1+d(x)) \vee 1)^{-1}}{\sqrt{\left|B\left(x, t^{1 / 2}\right)\right|}} \frac{(\sqrt{t} /(1+d(y)) \vee 1)^{-1}}{\sqrt{\left|B\left(y, t^{1 / 2}\right)\right|}} e^{-c d(x, y)^{2} / t} .
$$

This together with the standard Gaussian bounds for $G$ ([LY]), immediately shows

$$
\begin{gathered}
G_{V}(x, t ; y, 0) \leq C\left[\frac{(\sqrt{t} /(1+d(x)) \vee 1)^{-1}}{\sqrt{\left|B\left(x, t^{1 / 2}\right)\right|}} \frac{(\sqrt{t} /(1+d(y)) \vee 1)^{-1}}{\sqrt{\left|B\left(y, t^{1 / 2}\right)\right|}} e^{-c d(x, y)^{2} / t}\right]^{1 / \lambda} \\
\times\left[\frac{1}{\sqrt{\left|B\left(x, t^{1 / 2}\right)\right|} \sqrt{\left|B\left(y, t^{1 / 2}\right)\right|}} e^{-c d(x, y)^{2} / t}\right]^{1-(1 / \lambda)}
\end{gathered}
$$

It follows that

$$
G_{V}(x, t ; y, 0) \leq C \frac{(\sqrt{t} /(1+d(y)) \vee 1)^{-a / a_{0}}}{\sqrt{\left|B\left(x, t^{1 / 2}\right)\right|}} \frac{(\sqrt{t} /(1+d(y)) \vee 1)^{-a / a_{0}}}{\sqrt{\left|B\left(y, t^{1 / 2}\right)\right|}} e^{-c d(x, y)^{2} / t}
$$


for all $x, y$ and $t>0$. Here $a_{0}=64(n+4)^{2}$. The proposition follows.

In the following we use the heat kernel bounds in Proposition 2.1 to prove a refined mean value formula.

LEMMA 2.2. (refined mean value formula) Let $u$ be a nonnegative sub-solution to the equation $\Delta u-V u-u_{t}=0$ in $Q_{2 r}(x, t) \subset \mathbf{M} \times(0, \infty)$. Here $\mathbf{M}$ is as in Proposition 2.1.

Suppose, for a given $a>0$,

$$
V(x) \geq \frac{a}{1+d^{2}(x)}
$$

Then for $C>0$ and $\alpha=\alpha(a)$ in Proposition 2.1,

$$
u^{2}(x, t) \leq \frac{C[\max \{r /(1+d(x)), 1\}]^{-2 \alpha}}{\left|Q_{r}(x, t)\right|} \int_{Q_{r}(x, t)} u^{2}(y, s) d y d s .
$$

Proof. We select a cut-off function $\eta \in C_{0}^{\infty}\left(Q_{1.5 r}(x, t)\right)$ such that $\eta=1$ in $Q_{r}(x, t)$, $0 \leq \eta \leq 1$ everywhere. We also require that $|\nabla \eta| \leq C / r,|\Delta \eta| \leq C / r^{2},\left|\eta_{t}\right| \leq C / r^{2}$. Note that $\eta u$ satisfies

$$
\left\{\begin{array}{l}
\Delta(\eta u)-V \eta u-(\eta u)_{t} \geq u \Delta \eta+2 \nabla \eta \nabla u-u \eta_{t} \equiv f \\
\eta u(y, s)=0, \quad(y, s) \in \partial B(x, 1.5 r) \times\left[t-(1.5 r)^{2}, t\right] \\
\eta u\left(y, t-(1.5 r)^{2}\right)=0
\end{array}\right.
$$

Let $G_{q}$ be the Green's function of $\Delta u-V u-u_{t}=0$ in the cube $Q_{1.5 r}(x, t)$. Then

$$
u(x, t) \leq-\int_{Q_{1.5 r}(x, t)} G_{q}(x, t ; y, s) f(y, s) d y d s .
$$

By the maximum principle, $G_{q} \leq G_{V}$, the heat kernel of $\Delta-V u-u_{t}=0$ in the full space. Hence

$u(x, t)^{2} \leq C \int_{Q_{1.5 r}(x, t)-Q_{r}(x, t)} G_{V}^{2}(x, t ; y, s) d y d s \int_{Q_{1.5 r}(x, t)-Q_{r}(x, t)}\left(\frac{1}{r^{4}} u^{2}+\frac{1}{r^{2}}|\nabla u|^{2}\right) d y d s$.

Here we have used Hölder's inequality and the fact that $\nabla \eta=0, \eta_{t}=0$ in $Q_{r}(x, t)$.

Since $\Delta u-u_{t} \geq V u \geq 0$, it is well known that

$$
\int_{Q_{1.5 r}(x, t)-Q_{r}(x, t)}|\nabla u|^{2} d y d s \leq \frac{C}{r^{2}} \int_{Q_{2 r}(x, t)} u^{2} d y d s .
$$

Combing the last two inequalities, we reach

$$
u(x, t)^{2} \leq \frac{C}{r^{4}} \int_{Q_{1.5 r}(x, t)-Q_{r}(x, t)} G_{V}^{2}(x, t ; y, s) d y d s \int_{Q_{2 r}(x, t)} u^{2} d y d s .
$$

Now we use Proposition 2.1. Denoting $t-s$ by $l$, we obtain

$$
G_{V}(x, t ; y, s)=G_{V}(x, l ; y, 0) \leq c_{1} \frac{w(x, l)}{\left|B\left(x, l^{1 / 2}\right)\right|} e^{-c_{2} d(x, y)^{2} / l} .
$$


Here $w(x, l)=\left[\max \left\{\frac{l^{1 / 2}}{1+d(x)}, 1\right\}\right]^{-\alpha}$ with $\alpha=\alpha(a)>0$. Notice that

$$
\left[\frac{l^{1 / 2}}{1+d(x)}\right]^{-\alpha} e^{-c_{2} d(x, y)^{2} / l} \leq\left[\frac{1+d(x)}{\sqrt{l}}\right]^{\alpha} e^{-c_{2} d(x, y)^{2} / l} \leq\left[\frac{1+d(x)}{d(x, y)}\right]^{\alpha} e^{-c_{2} d(x, y)^{2} /(2 l)}
$$

When $(y, s) \in Q_{1.5 r}(x, t)-Q_{r}(x, t)$ and $t-r^{2} \leq s \leq t$, we have $d(x, y) \geq r$. Hence

$$
G_{V}(x, t ; y, s) \leq c_{1} \frac{\left(\left[\frac{r}{1+d(x)}\right) \vee 1\right]^{-\alpha}}{\left|B\left(x, l^{1 / 2}\right)\right|} e^{-c_{2} d(x, y)^{2} /(2 l)}
$$

By standard arguments using the doubling condition of balls, we know that

$$
G_{V}(x, t ; y, s) \leq c_{1} \frac{\left(\left[\frac{r}{1+d(x)}\right) \vee 1\right]^{-\alpha}}{|B(x, d(x, y))|} \leq c_{1} \frac{\left(\left[\frac{r}{1+d(x)}\right) \vee 1\right]^{-\alpha}}{|B(x, r)|}
$$

when $(y, s) \in Q_{1.5 r}(x, t)-Q_{r}(x, t)$ and $t-r^{2} \leq s \leq t$.

On the other hand, when $(y, s) \in Q_{1.5 r}(x, t)-Q_{r}(x, t)$ and $t-(1.5 r)^{2} \leq s \leq t-r^{2}$, we have $l=t-s \geq r^{2}$. Therefore

$$
G_{V}(x, t ; y, s) \leq c_{1} \frac{w(x, l)}{\left|B\left(x, l^{1 / 2}\right)\right|} e^{-c_{2} d(x, y)^{2} / l} \leq c \frac{\left(\left[\frac{r}{1+d(x)}\right) \vee 1\right]^{-\alpha}}{|B(x, r)|}
$$

Therefore

$$
G_{V}(x, t ; y, s) \leq c \frac{\left(\left[\frac{r}{1+d(x)}\right) \vee 1\right]^{-\alpha}}{|B(x, r)|}
$$

for all $(y, s) \in Q_{1.5 r}(x, t)-Q_{r}(x, t)$. Substituting this to (2.7), we have

$$
u(x, t)^{2} \leq\left[\frac{r}{1+d(x)} \vee 1\right]^{-2 \alpha} \frac{C}{r^{2}|B(x, r)|} \int_{Q_{2 r}(x, t)} u^{2} d y d s
$$

Proof of Theorem 1.1. Let $G=G(x, t ; y, 0)$ be the heat kernel of $\Delta$ in M. Fixing $x$ and $y$ and taking $r=\sqrt{t} / 2$, then $u(z, \tau) \equiv G(z, \tau ; y, 0)$ is a caloric function in $Q_{r}(x, t)=B(x, r) \times[3 t / 4, t]$. By Bochner's formula, it is well known that

$$
\Delta|\nabla u(z, \tau)|-\frac{R_{i j} u_{i}(z, \tau) u_{j}(z, \tau)}{|\nabla u|^{2}}|\nabla u(z, \tau)|-\partial_{\tau}|\nabla u(z, \tau)| \geq 0
$$

Here $R_{i j}$ is the Ricci curvature, in local coordinates. Indeed, in local orthonormal frames,

$$
\begin{aligned}
\Delta|\nabla u| & =\Delta\left(\Sigma_{i} u_{i}^{2}\right)^{1 / 2}=\Sigma_{j}\left(\left(\Sigma_{i} u_{i}^{2}\right)^{1 / 2}\right)_{j j}=\Sigma_{j}\left[\frac{\Sigma_{i} u_{i} u_{i j}}{\left(\Sigma_{i} u_{i}^{2}\right)^{1 / 2}}\right]_{j} \\
& =\frac{\Sigma_{i j} u_{i j}^{2}}{\left(\Sigma_{i} u_{i}^{2}\right)^{1 / 2}}+\frac{\Sigma_{i j} u_{i} u_{i j j}}{\left(\Sigma_{i} u_{i}^{2}\right)^{1 / 2}}-\frac{\Sigma_{j}\left(\Sigma_{i} u_{i} u_{i j}\right)^{2}}{\left(\Sigma_{i} u_{i}^{2}\right)^{3 / 2}} \geq \frac{R_{i j} u_{i} u_{j}}{|\nabla u|}+\partial_{\tau}|\nabla u(z, \tau)| .
\end{aligned}
$$

By our assumption on the Ricci curvature, the above can be written as

$$
\Delta|\nabla u(z, \tau)|-V(z)|\nabla u(z, \tau)|-\partial_{\tau}|\nabla u(z, \tau)| \geq 0 .
$$


where

$$
V(z) \geq \frac{a}{1+d(z)^{2}}, \quad a>0 .
$$

By Lemma 2.2, the refined mean value formula,

$$
|\nabla u(x, t)|^{2} \leq\left(\frac{1+r}{1+d(x)} \vee 1\right)^{-2 \alpha} \frac{C}{\left|Q_{r}(x, t)\right|} \int_{Q_{r}(x, t)}|\nabla u(z, \tau)|^{2} d z
$$

From [LY], for any $c>0$,

$$
|\nabla u(z, \tau)|=\left|\nabla_{z} G(z, \tau ; y, 0)\right| \leq \frac{C}{\sqrt{\tau}|B(z, \tau)|} \exp \left(-\frac{d(z, y)^{2}}{(4+c) \tau}\right) .
$$

When $(z, \tau) \in B(x, r) \times[3 t / 4, t]$, we have $d(z, y) \geq d(x, y)-d(x, z) \geq d(x, y)-(\sqrt{t} / 2)$.

Also, by the doubling condition of geodesic balls, one has $|B(z, r)| \sim|B(x, r)|$. Therefore

$$
|\nabla u(z, \tau)| \leq \frac{C}{\sqrt{t}|B(x, \sqrt{t})|} \exp \left(-\frac{d(x, y)^{2}}{(4+c) t}\right)
$$

Substituting (2.10) to (2.9), we obtain

$$
|\nabla u(x, t)|^{2} \leq\left(\frac{1+r}{1+d(x)} \vee 1\right)^{-2 \alpha} \frac{C}{\left|Q_{r}(x, t)\right|} \int_{Q_{r}(x, t)}\left(\frac{1}{\sqrt{t}|B(x, \sqrt{t})|} e^{-d(x, y)^{2} /(4+c) t}\right)^{2} d z
$$

Hence

$$
|\nabla u(x, t)| \leq C\left(\frac{1+\sqrt{t}}{1+d(x)} \vee 1\right)^{-\alpha} \frac{1}{\sqrt{t}|B(x, \sqrt{t})|} e^{-d(x, y)^{2} /(4+c) t}
$$

Next we prove some technical estimates on the integral of the gradient of the heat kernel. This will be essential for the proof of Theorem 1.2.

First we need a lower estimate for the volume of geodesic balls that generalizes Theorem 1.4.1 in [SY]. The result is known, see [Wa] Lemma 2 e.g. But for completeness, we give a proof here which follows the same idea as the above quoted Theorem.

Proposition 2.2. Let $\mathbf{M}$ be a noncompact Riemannian manifold with nonnegative Ricci curvature. For any $p \in \mathbf{M}, r>0$, and $k \geq 1$, there exists a $C>0$ independent of $p, r$, and $k$ such that $|B(p, k r)| \geq C k|B(p, r)|$.

Proof. It is clear that we can assume that $k \geq 2$. Pick a point $x_{0}$ such that $d\left(p, x_{0}\right)=k r$ and, for $x \in \mathbf{M}$, write $\rho=d\left(x, x_{0}\right)$. Similar to Theorem 1.4.1 in [SY], we construct a Lipschitz function $\psi$ so that

$$
\psi(t)=\left\{\begin{array}{l}
1, \quad 0 \leq t \leq(k-1) r \\
\psi^{\prime}(t)=-1 /(2 r), \quad(k-1) r<t<(k+1) r \\
0, \quad t \geq(k+1) r .
\end{array}\right.
$$

Define $\phi=\psi(\rho(x))$, a Lipschitz function in $\mathbf{M}$. Note that the Ricci curvature is nonnegative. As in [SY], we have, in distribution sense,

$$
\int_{\mathbf{M}} \phi(x) \Delta \rho^{2}(x) \leq 2 n \int_{\mathbf{M}} \phi(x) .
$$


On the other hand, one has, in distribution sense

$$
\begin{aligned}
\int_{\mathbf{M}} & \phi(x) \Delta \rho^{2}(x)=-\int_{\mathbf{M}} \nabla \phi(x) \nabla \rho^{2}(x) \\
& =-2 \int_{B\left(x_{0},(k+1) r\right)-B\left(x_{0},(k-1) r\right)} \psi^{\prime}(\rho) \rho|\nabla \rho|^{2}=\frac{1}{r} \int_{B\left(x_{0},(k+1) r\right)-B\left(x_{0},(k-1) r\right)} \rho \\
& \geq \frac{(k-1) r}{r}\left|B\left(x_{0},(k+1) r\right)-B\left(x_{0},(k-1) r\right)\right| .
\end{aligned}
$$

Since $B(p, r)$ is contained in $B\left(x_{0},(k+1) r\right)-B\left(x_{0},(k-1) r\right)$, the above shows

$$
\int_{\mathbf{M}} \phi(x) \Delta \rho^{2}(x) \geq(k-1)|B(x, R)| \text {. }
$$

Combining this the (2.11), we see that

$$
2 n|B(x,(k+1) R)| \geq 2 n \int_{\mathbf{M}} \phi \geq \int_{\mathbf{M}} \phi(x) \Delta \rho^{2}(x) \geq(k-1)|B(x, R)| .
$$

By now, the proposition is an immediate consequence of the doubling property of geodesic balls.

The following is a technical gradient estimate for the integral of the heat kernels of parabolic manifolds. This will replace the gradient bound of the 'Green's function' of the Laplacian, which may or may not exists in this case.

Proposition 2.3. Let $\mathbf{M}$ be a noncompact Riemannian manifold such that $\operatorname{Ric}(x) \geq a /\left(1+d(x)^{2}\right)$ for some $a>0$. Let $G$ be the heat kernel of the Laplacian. Then, for the same $\alpha$ as before

$$
\int_{0}^{\infty}\left|\nabla_{x} G(x, t ; y, 0)\right| d t \leq\left\{\begin{array}{l}
C\left(\frac{d(x, y)}{1+d(x)}\right)^{-\alpha} \frac{d(x, y)}{|B(x, d(x, y))|}, \quad d(x) \leq d(x, y) ; \\
\frac{d(x, y)}{|B(x, d(x, y))|}\left[\ln \left(e+\frac{d(x)}{d(x, y)}\right)+\left(\ln \frac{1}{d(x)} \vee 0\right)\right], \quad d(x) \geq d(x, y) .
\end{array}\right.
$$

In particular, for all $x$ and $y$ in $\mathbf{M}$,

$$
\int_{0}^{\infty}\left|\nabla_{x} G(x, t ; y, 0)\right| d t \leq C\left(\frac{d(x, y)}{1+d(x)}\right)^{-\alpha} \frac{d(x, y)}{|B(x, d(x, y))|}
$$

Proof. Let $G$ be the heat kernel of $\Delta$, i.e. the fundamental solution of $\Delta u-u_{t}=0$ in $\mathbf{M}$. By [LY] we have, for all $x, y \in \mathbf{M}$ and $t>0$,

$$
\left|\nabla_{x} G(x, t ; y, 0)\right| \leq \frac{c_{1}}{\sqrt{t}|B(x, \sqrt{t})|} e^{-c_{2} d(x, y)^{2} / t} .
$$

By the well known Theorem of Calabi and Yau, one has $|B(x, \sqrt{t})| \geq C_{x} t, t \geq 1$. So the above may not be integrable for any open manifold with nonnegative Ricci curvature. However by Theorem 1.1, we can pick up some extra decay in time under our lower bound on the Ricci. In fact

$$
\left|\nabla_{x} G(x, t ; y, 0)\right| \leq\left(\frac{1+\sqrt{t}}{1+d(x)} \vee 1\right)^{-\alpha} \frac{C}{\sqrt{t}|B(x, \sqrt{t})|} e^{-c_{2} d(x, y)^{2} / t} .
$$

Here $\alpha>0$ is given as in Theorem 1.1. The extra decay in time allows us to integrate the gradient of the heat kernel. 
By (2.13)

$$
\begin{aligned}
\int_{0}^{\infty} & \left|\nabla_{x} G(x, t ; y, 0)\right| d t \\
\leq & \int_{0}^{\infty}\left(\frac{1+\sqrt{t}}{1+d(x)} \vee 1\right)^{-\alpha} \frac{C}{\sqrt{t}|B(x, \sqrt{t})|} e^{-c_{2} d(x, y)^{2} / t} d t \\
= & C \int_{0}^{d(x)^{2}} \frac{C}{\sqrt{t}|B(x, \sqrt{t})|} e^{-c_{2} d(x, y)^{2} / t} d t \\
& \quad+C \int_{d(x)^{2}}^{\infty}\left(\frac{1+\sqrt{t}}{1+d(x)} \vee 1\right)^{-\alpha} \frac{e^{-c_{2} d(x, y)^{2} / t}}{\sqrt{t}|B(x, \sqrt{t})|} d t \\
\equiv & =C I_{1}+C I_{2} .
\end{aligned}
$$

Let us estimate $I_{1}$ first. We need to distinguish two cases.

Case $1.1 d(x) \leq d(x, y)$.

By the doubling condition of the balls, it is well known that

$$
\frac{1}{\sqrt{t}|B(x, \sqrt{t})|} e^{-c_{2} d(x, y)^{2} / t} d t \leq \frac{C}{d(x, y)|B(x, d(x, y))|} e^{-c_{2} d(x, y)^{2} /(2 t)} .
$$

Hence

$$
I_{1} \leq \frac{C}{d(x, y)|B(x, d(x, y))|} \int_{0}^{d(x)^{2}} d t \leq \frac{C d(x)^{2}}{d(x, y)|B(x, d(x, y))|}
$$

i.e.

$$
I_{1} \leq \frac{d(x)}{d(x, y)} \frac{C d(x, y)}{|B(x, d(x, y))|}, \quad d(x) \leq d(x, y) .
$$

This finishes case 1.1.

Case 1.2. $d(x) \geq d(x, y)$.

Then

$$
I_{1}=\int_{0}^{d(x, y)^{2}} \frac{C}{\sqrt{t}|B(x, \sqrt{t})|} e^{-c_{2} d(x, y)^{2} / t} d t+\int_{d(x, y)^{2}}^{d(x)^{2}} \frac{C}{\sqrt{t}|B(x, \sqrt{t})|} e^{-c_{2} d(x, y)^{2} / t} d t .
$$

Applying (2.16) on the first integral on the left hand side in the above, we have

$$
I_{1} \leq \frac{C d(x, y)}{|B(x, d(x, y))|}+\int_{d(x, y)^{2}}^{d(x)^{2}} \frac{C}{\sqrt{t}|B(x, \sqrt{t})|} e^{-c_{2} d(x, y)^{2} / t} d t .
$$

When $t \geq d(x, y)^{2}$, by Proposition 2.2,

$$
|B(x, \sqrt{t})| \geq C \frac{\sqrt{t}}{d(x, y)}|B(x, d(x, y))| .
$$

A Combination of the last two inequalities yield

$$
\begin{aligned}
I_{1} & \leq \frac{C d(x, y)}{|B(x, d(x, y))|} \int_{d(x, y)^{2}}^{d(x)^{2}} \frac{1}{t} e^{-c_{2} d(x, y)^{2} / t} d t \\
& \leq \frac{C d(x, y)}{|B(x, d(x, y))|} \ln (d(x) / d(x, y)) .
\end{aligned}
$$


This finishes case 1.2.

By (2.16) and (2.17), we deduce

$$
I_{1} \leq\left(\frac{d(x, y)}{d(x)} \vee 1\right)^{-1} \frac{C d(x, y)}{|B(x, d(x, y))|} \ln \left[e+\frac{d(x)}{d(x, y)}\right]
$$

for all $x$ and $y$.

Next we estimate $I_{2}$. We need to treat two cases again.

Case 2.1. $d(x) \leq d(x, y)$.

Then

$$
\begin{aligned}
I_{2}= & C(1+d(x))^{\alpha} \int_{d(x)^{2}}^{\infty} \frac{e^{-c_{2} d(x, y)^{2} / t}}{(1+\sqrt{t})^{\alpha} \sqrt{t}|B(x, \sqrt{t})|} \\
= & C(1+d(x))^{\alpha} \int_{d(x)^{2}}^{d(x, y)^{2}} \frac{e^{-c_{2} d(x, y)^{2} / t}}{(1+\sqrt{t})^{\alpha} \sqrt{t}|B(x, \sqrt{t})|} \\
& \quad+C(1+d(x))^{\alpha} \int_{d(x, y)^{2}}^{\infty} \frac{e^{-c_{2} d(x, y)^{2} / t}}{(1+\sqrt{t})^{\alpha} \sqrt{t}|B(x, \sqrt{t})|} d t \\
& =C(1+d(x))^{\alpha} J_{1}+C(1+d(x))^{\alpha} J_{2} .
\end{aligned}
$$

To estimate $J_{1}$, let us note

$$
\frac{e^{-c_{2} d(x, y)^{2} / t}}{(1+\sqrt{t})^{\alpha} \sqrt{t}|B(x, \sqrt{t})|} \leq \frac{e^{-c_{2} d(x, y)^{2} /(2 t)}}{(1+d(x, y))^{\alpha} d(x, y)|B(x, d(x, y))|} .
$$

This is due to the doubling property of the geodesic balls again. Hence

$$
J_{1} \leq \frac{d(x, y)^{2}-d(x)^{2}}{(1+d(x, y))^{\alpha} d(x, y)|B(x, d(x, y))|}
$$

i.e.

$$
J_{1} \leq \frac{d(x, y)}{(1+d(x, y))^{\alpha}|B(x, d(x, y))|}
$$

To estimate $J_{2}$, we use Proposition 2.2 again. For $t \geq d(x, y)^{2}$,

$$
|B(x, \sqrt{t})| \geq C \frac{\sqrt{t}}{d(x, y)}|B(x, d(x, y))| .
$$

So

$$
J_{2} \leq \frac{d(x, y)}{|B(x, d(x, y))|} \int_{d(x, y)^{2}}^{\infty} \frac{1}{(1+\sqrt{t})^{\alpha} t} d t
$$

This shows

$$
J_{2} \leq \frac{d(x, y)}{d(x, y)^{\alpha}|B(x, d(x, y))|}
$$

$\mathrm{By}(2.19),(2.20)$ and $(2.21)$, we have, when $d(x) \leq d(x, y)$,

$$
I_{2} \leq C\left(\frac{1+d(x)}{d(x, y)}\right)^{\alpha} \frac{d(x, y)}{|B(x, d(x, y))|}
$$


This ends case 2.1.

Finally let us estimate $I_{2}$ in

Case 2.2. $d(x) \geq d(x, y)$.

From the definition of $I_{2}$ in (2.14) we know that

$$
\begin{aligned}
I_{2} & =\int_{d(x)^{2}}^{\infty}\left(\frac{1+\sqrt{t}}{1+d(x)} \vee 1\right)^{-\alpha} \frac{e^{-c_{2} d(x, y)^{2} / t}}{\sqrt{t}|B(x, \sqrt{t})|} d t \\
& \leq C(1+d(x))^{\alpha} \int_{d(x)^{2}}^{\infty} \frac{e^{-c_{2} d(x, y)^{2} / t}}{(1+\sqrt{t})^{\alpha} \sqrt{t}|B(x, \sqrt{t})|} d t .
\end{aligned}
$$

Using the inequality

$$
|B(x, \sqrt{t})| \geq C \frac{\sqrt{t}}{d(x)}|B(x, d(x))|
$$

for $t \geq d(x)^{2}$ (by Proposition 2.2), we have

$$
I_{2} \leq C(1+d(x))^{\alpha} \frac{d(x)}{|B(x, d(x))|} \int_{d(x)^{2}}^{\infty} \frac{1}{(1+\sqrt{t})^{\alpha} t} d t .
$$

Therefore

$$
I_{2} \leq C(1+d(x))^{\alpha} \frac{d(x)}{|B(x, d(x))|} \frac{1}{d(x)^{\alpha}} .
$$

By Proposition 2.2 again, when $d(x) \geq d(x, y)$,

$$
|B(x, d(x))| \geq C \frac{d(x)}{d(x, y)}|B(x, d(x, y))| .
$$

This shows

$$
I_{2} \leq\left(\frac{1+d(x)}{d(x)}\right)^{\alpha} \frac{d(x, y)}{|B(x, d(x, y))|} \leq \frac{d(x, y)}{|B(x, d(x, y))|}
$$

when $d(x) \geq d(x, y)$ and $d(x) \geq 1$.

When $d(x) \leq 1$, it is easy to see that $\int_{d(x)^{2}}^{\infty} \frac{1}{(1+\sqrt{t})^{\alpha} t} d t \leq C\left(1+\ln \frac{1}{d(x)}\right)$. Hence

$$
I_{2} \leq C \frac{d(x, y)}{|B(x, d(x, y))|}\left(1+\ln \frac{1}{d(x)}\right)
$$

when $1 \geq d(x) \geq d(x, y)$. Therefore

$$
I_{2} \leq C \frac{d(x, y)}{|B(x, d(x, y))|}\left[1+\left(\ln \frac{1}{d(x)} \vee 0\right)\right], \quad d(x) \geq d(x, y)
$$

This concludes case 2.2.

Combining this with (2.22), we know that, for all $x$ and $y$,

$$
I_{2} \leq\left\{\begin{array}{l}
C\left(\frac{1+d(x)}{d(x, y)}\right)^{\alpha} \frac{d(x, y)}{|B(x, d(x, y))|}, \quad d(x) \leq d(x, y) \\
C \frac{d(x, y)}{|B(x, d(x, y))|}\left[1+\left(\ln \frac{1}{d(x)} \vee 0\right)\right], \quad d(x) \geq d(x, y) .
\end{array}\right.
$$


By (2.14), (2.18) and (2.23), we have finally proved

$$
\int_{0}^{\infty}|\nabla G(x, t ; y, 0)| d t \leq\left\{\begin{array}{l}
C\left(\frac{d(x, y)}{1+d(x)}\right)^{-\alpha} \frac{d(x, y)}{\mid B(x, d(x, y))}, \quad d(x) \leq d(x, y) \\
\frac{d(x, y)}{|B(x, d(x, y))|}\left[\ln \left(e+\frac{d(x)}{d(x, y)}\right)+\left(\ln \frac{1}{d(x)} \vee 0\right)\right], \quad d(x) \geq d(x, y) .
\end{array}\right.
$$

Since, when $d(x) \geq d(x, y)$,

$$
\left[\ln \left(e+\frac{d(x)}{d(x, y)}\right)+\left(\ln \frac{1}{d(x)} \vee 0\right)\right] \leq C\left(\frac{1+d(x)}{d(x, y)}\right)^{\alpha},
$$

the second inequality in the statement of the proposition also holds.

3. Solvability of $\Delta u=f$ and $\sqrt{-1} \partial \bar{\partial} u=f$ when $\operatorname{Ric}(x) \geq a /\left(1+d(x)^{2}\right)$. We will prove Theorem 1.2 first. The basic approach is to combine the integration arguments in [NST] with a heat kernel approach using the refined gradient bounds. This combination will allow us to treat long range $f$ without imposing pointwise bounds. It will also allow us to treat parabolic and nonparabolic manifolds at the same time. In [NST], the parabolic case was handled by multiplying $\mathbf{R}^{4}$ on the given manifold to make it nonparabolic. We are not able to use this approach since the current solutions may grow in the order of $(1+d(x))^{1+\epsilon}$.

We present a

Proof of Theorem 1.2 part (a). We divide the proof into several steps.

Step 1 . Let $G$ be the heat kernel of $\Delta$ in M. Given $f$ as in the statement of the theorem, we construct a function

$$
u(x, t)=\int_{0}^{t} \int_{\mathbf{M}}[G(0, s ; y, 0)-G(x, s ; y, 0)] f(y) d y d s .
$$

Clearly $u$ is well defined and satisfies

$$
\Delta u-u_{t}=f(x)-\int_{\mathbf{M}} G(0, t ; y, 0) f(y) d y .
$$

We are going to show that $u\left(., t_{j}\right)$ converges to a desired solution of (1.1). Here $\left\{t_{j}\right\}$ is a sequence diverging to $\infty$.

Let $l$ be a minimum geodesic connecting 0 and $x$, parameterized by arc-length. Then

$$
\begin{aligned}
G(0, s ; y, 0)-G(x, s ; y, 0) & =-\int_{0}^{r} \partial_{\tau} G(l(\tau), s ; y, 0) d \tau \\
& =-\int_{0}^{r} g\left(\nabla G(l(\tau), s ; y, 0), l^{\prime}(\tau)\right) d \tau
\end{aligned}
$$

Here $r=d(x)$. Therefore

$$
|u(x, t)| \leq \int_{0}^{t} \int_{\mathbf{M}} \int_{0}^{r}|\nabla G(l(\tau), s ; y, 0) \| f(y)| d \tau d y d s .
$$

Switching the order of integration, we see that there exists a point $z \in B(0, r)$ such that

$$
|u(x, t)| \leq r \int_{0}^{t} \int_{\mathbf{M}}\left|\nabla_{z} G(z, s ; y, 0)\right||f(y)| d y d s
$$


Therefore

$$
|u(x, t)| \leq r \int_{\mathrm{M}} \int_{0}^{\infty}\left|\nabla_{z} G(z, s ; y, 0)\right| d s|f(y)| d y .
$$

We split the integral on the last inequality to get

$$
\begin{aligned}
|u(x, t)| \leq r & \int_{d(y) \geq 2 d(x)} \int_{0}^{\infty}\left|\nabla_{z} G(z, s ; y, 0)\right| d s|f(y)| d y \\
& \quad+r \int_{d(y) \leq 2 d(x)} \int_{0}^{\infty}\left|\nabla_{z} G(z, s ; y, 0)\right| d s|f(y)| d y \\
\equiv & I I I
\end{aligned}
$$

Step 2. Here we will estimate $I$.

Note that $d(y) \geq 2 d(x)$ implies $d(x, y) \geq d(x)$. Also $d(z) \leq d(x)$. By Proposition 2.3 ,

$$
\int_{0}^{\infty}\left|\nabla_{z} G(z, s ; y, 0)\right| d s \leq C\left(\frac{d(z, y)}{1+d(z)}\right)^{-\alpha} \frac{d(z, y)}{|B(z, d(z, y))|}
$$

By the doubling condition of geodesic balls,

$$
\int_{0}^{\infty}\left|\nabla_{z} G(z, s ; y, 0)\right| d s \leq C\left(\frac{d(x, y)}{1+d(x)}\right)^{-\alpha} \frac{d(x, y)}{|B(x, d(x, y))|}
$$

Hence

$$
I \leq C(1+d(x))^{1+\alpha} \int_{d(y) \geq 2 d(x)} \frac{d(x, y)}{d(x, y)^{\alpha}|B(x, d(x, y))|}|f(y)| d y .
$$

Given any large $R>0$, let us write

$$
I_{R} \equiv(1+d(x))^{1+\alpha} \int_{B(0, R)-B(0,2 r)} \frac{d(x, y)}{|B(x, d(x, y))| d(x, y)^{\alpha}}|f(y)| d y .
$$

Since $d(x, y) \geq r=d(x)$, we can write the above as

$$
\begin{aligned}
I_{R} & \leq C(1+d(x))^{1+\alpha} \int_{B(0, R)-B(0,2 r)} \frac{d(0, y)}{|B(0, d(y))| d(y)^{\alpha}}|f(y)| d y \\
& \leq C(1+d(x))^{1+\alpha} \int_{2 r}^{R} \frac{t^{1-\alpha}}{|B(0, t)|}\left(\int_{\partial B(0, t)}|f(y)|\right) d t .
\end{aligned}
$$

Note that

$$
\partial_{t} \int_{B(0, t)}|f(y)|=\int_{\partial B(0, t)}|f(y)| .
$$

Using integration by parts, we obtain

$$
\begin{aligned}
I_{R} \leq C & (1+d(x))^{1+\alpha} \frac{R^{1-\alpha}}{|B(0, R)|} \int_{B(0, R)}|f(y)| d y \\
& -C(1+d(x))^{1+\alpha} \int_{2 r}^{R}\left[\frac{1-\alpha}{t^{\alpha}|B(0, t)|}\left(\int_{B(0, t)}|f|\right) d t\right. \\
& +C(1+d(x))^{1+\alpha} \int_{2 r}^{R}\left[\frac{t^{1-\alpha}|\partial B(0, t)|}{|B(0, t)|^{2}}\left(\int_{B(0, t)}|f|\right) d t .\right.
\end{aligned}
$$


Since the Ricci curvature is nonnegative, it is known that (see [Wa] e.g.),

$$
\frac{|\partial B(0, t)|}{|B(0, t)|} \leq \frac{C}{t}
$$

Therefore the above implies

$$
\begin{aligned}
I_{R} \leq C & (1+d(x))^{1+\alpha} \frac{R^{1-\alpha}}{|B(0, R)|} \int_{B(0,3 R)}|f(y)| d y \\
& +C(1+d(x))^{1+\alpha} \int_{2 r}^{R}\left[\frac{1}{t^{\alpha}|B(0, t)|}\left(\int_{B(0, t)}|f|\right) d t .\right.
\end{aligned}
$$

This shows

$$
I_{R} \leq C(1+d(x))^{1+\alpha}\left(\frac{R}{|B(0, R)| R^{\alpha}} \int_{B(0, R)}|f(y)| d y+\int_{2 r}^{R} \frac{1}{|B(0, t)| t^{\alpha}} \int_{B(0, t)}|f(y)| d y d t\right) .
$$

Under our assumptions on $f$,

$$
\int_{2 r}^{\infty} \frac{1}{|B(0, t)| t^{\alpha}} \int_{B(0, t)}|f(y)| d y d t<\infty
$$

Denoting

$$
K_{\alpha}(R) \equiv \frac{1}{|B(0, R)| R^{\alpha}} \int_{B(0, R)}|f(y)| d y,
$$

it is clear that $K_{\alpha}(\lambda R) \geq C K_{\alpha}(R)$ for $\lambda \in[1,2]$. Using this and the assumption that $\int_{1}^{\infty} K_{\alpha}(r) d r<\infty$, by elementary arguments, we see that

$$
R K_{\alpha}(R)=\frac{R}{|B(0, R)| R^{\alpha}} \int_{B(0, R)}|f(y)| d y \rightarrow 0, \quad R \rightarrow \infty .
$$

Hence we have

$$
I_{R} \leq C(1+d(x))^{1+\alpha}
$$

Therefore

$$
I \leq C(1+d(x))^{1+\alpha}
$$

This completes step 1.

Step 2. Here we will estimate $I I$.

By the second inequality in Proposition 2.3, we have

$$
\int_{0}^{\infty}\left|\nabla_{z} G(z, s ; y, 0)\right| d s \leq C\left(\frac{d(z, y)}{1+d(z)}\right)^{-\alpha} \frac{d(z, y)}{|B(z, d(z, y))|}
$$

for all $y, z$.

This shows

$$
\begin{aligned}
I I & \leq C r \int_{B(0,2 r)} \int_{0}^{\infty}\left|\nabla_{z} G(z, s ; y, 0)\right| d s|f(y)| d y \\
& \leq C r \int_{B(0,2 r)}\left(\frac{d(z, y)}{1+d(z)}\right)^{-\alpha} \frac{d(z, y)}{|B(z, d(z, y))|}|f(y)| d y .
\end{aligned}
$$


Since $d(z) \leq d(x)$, the above implies

$$
I I \leq C r \int_{B(z, 3 r)}\left(\frac{d(z, y)}{1+d(z)}\right)^{-\alpha} \frac{d(z, y)}{|B(z, d(z, y))|}|f(y)| d y .
$$

Now we can follow the arguments from (3.6) to (3.8) to get

$$
\begin{aligned}
I I & \leq C(1+d(x))^{1+\alpha} \int_{B(z, 3 r)} \frac{d(z, y)}{|B(z, d(y, z))| d(y, z)^{\alpha}}|f(y)| d y \\
& \leq C(1+d(x))^{1+\alpha} \int_{0}^{2 r} \frac{t^{1-\alpha}}{|B(z, t)|}\left(\int_{\partial B(z, t)}|f(y)|\right) d t .
\end{aligned}
$$

Note that

$$
\partial_{t} \int_{B(z, t)}|f(y)|=\int_{\partial B(z, t)}|f(y)| .
$$

Using integration by parts, we obtain

$$
\begin{aligned}
I I \leq C & (1+d(x))^{1+\alpha} \frac{r^{1-\alpha}}{|B(z, 3 r)|} \int_{B(z, 3 r)}|f(y)| d y \\
& -C(1+d(x))^{1+\alpha} \int_{0}^{3 r}\left[\frac{1-\alpha}{t^{\alpha}|B(z, t)|}\left(\int_{B(z, t)}|f|\right) d t\right. \\
& +C(1+d(x))^{1+\alpha} \int_{0}^{3 r}\left[\frac{t^{1-\alpha}|\partial B(z, t)|}{|B(z, t)|^{2}}\left(\int_{B(z, t)}|f|\right) d t .\right.
\end{aligned}
$$

Since the Ricci curvature is nonnegative, it is known that

$$
\frac{|\partial B(z, t)|}{|B(z, t)|} \leq \frac{C}{t}
$$

Therefore the above implies

$$
\begin{aligned}
I I \leq C & (1+d(x))^{1+\alpha} \frac{r^{1-\alpha}}{|B(z, 3 r)|} \int_{B(z, 3 r)}|f(y)| d y \\
& +C(1+d(x))^{1+\alpha} \int_{0}^{3 r}\left[\frac{1}{t^{\alpha}|B(z, t)|}\left(\int_{B(z, t)}|f|\right) d t .\right.
\end{aligned}
$$

Using the doubling condition again, we have

$$
I I \leq C(1+d(x))^{1+\alpha}\left(\frac{r^{1-\alpha}}{|B(0,4 r)|} \int_{B(0,4 r)}|f(y)| d y+\int_{0}^{3 r} \frac{1}{|B(z, t)| t^{\alpha}} \int_{B(z, t)}|f(y)| d y d t\right) .
$$

As before, by our assumption on $f$, this shows

$$
I I \leq C(1+d(x))^{1+\alpha} .
$$

Going back to (3.4), we have, for all $t \geq 1$,

$$
|u(x, t)| \leq I+I I \leq C(1+d(x))^{1+\alpha} .
$$


From (3.5) and (3.9) we also know that

$$
|u(x, t)| \leq C(1+d(x))^{1+\alpha} \sup _{z} \int_{\mathbf{M}} \frac{d(z, y)}{d(z, y)^{\alpha}|B(z, d(z, y))|}|f(y)| d y .
$$

Step 3. We show that $u\left(x, t_{j}\right)$ converges to a solution of (1.1). Here $t_{j}$ is a suitable subsequence diverging to $\infty$.

By standard parabolic theory, $u$ is locally Hölder continuous. So there exists a sequence $\left\{t_{j}\right\}$ so that $u\left(., t_{j}\right)$ converges to a function $u=u_{0}(x)$ as $t_{j} \rightarrow \infty$.

Next notice that

$$
u_{t}(x, t)=\int_{\mathbf{M}} G(0, t ; y, 0) f(y) d y-\int_{\mathbf{M}} G(x, t ; y, 0) f(y) d y .
$$

By our assumption, one can quickly show that

$$
u_{t}(x, t) \rightarrow 0
$$

as $t \rightarrow \infty$. In fact this has been proven in [Ni] Corollary 3.3. For completeness, we will just give a sketch to show that $\int_{\mathbf{M}} G(0, t ; y, 0) f(y) d y \rightarrow 0$ as $t \rightarrow \infty$ since the second term can be dealt with in the same way. By the standard Gaussian bounds,

$$
I(0, t) \equiv \int_{\mathbf{M}} G(0, t ; y, 0)|f(y)| d y \leq \int_{\mathbf{M}} \frac{c_{1}}{|B(0, \sqrt{t})|} e^{-c_{2} d(y)^{2} / t}|f(y)| d y .
$$

Using integration by part as in $[\mathrm{Ni}]$, one has

$$
\begin{aligned}
& I(0, t) \leq \frac{c_{1}}{|B(0, \sqrt{t})|} \int_{0}^{\infty} e^{-c_{2} r^{2} / t}\left(\int_{\partial B(0, r)}|f(y)|\right) d r \\
& \leq \frac{c_{1}}{|B(0, \sqrt{t})|} \int_{0}^{\sqrt{t}} e^{-c_{2} r^{2} / t}\left(\int_{\partial B(0, r)}|f(y)|\right) d r \\
& \quad+\frac{c_{1}}{|B(0, \sqrt{t})|} \int_{\sqrt{t}}^{\infty} e^{-c_{2} r^{2} / t}\left(\int_{\partial B(0, r)}|f(y)|\right) d r \\
& \leq \frac{c_{1}}{|B(0, \sqrt{t})|} \int_{0}^{\sqrt{t}}\left(\int_{\partial B(0, r)}|f(y)|\right) d r \\
& \quad+C \int_{\sqrt{t}}^{\infty} \frac{1}{|B(0, \sqrt{t})|} \int_{B(0, r)}|f(y)| d y \frac{r}{t} e^{-c_{2} r^{2} / t} d r .
\end{aligned}
$$

When $r \geq \sqrt{t}$, one has $|B(0, \sqrt{t})| \geq|B(0, r)|(\sqrt{t} / r)^{n}$ by volume comparison theorem. Hence, when $t \geq 1$,

$$
\begin{aligned}
I(0, t) \leq & \frac{c_{1}}{|B(0, \sqrt{t})|} \int_{0}^{\sqrt{t}}\left(\int_{\partial B(0, r)}|f(y)|\right) d r \\
& \quad+C \int_{\sqrt{t}}^{\infty} \frac{1}{|B(0, r)|} \int_{B(0, r)}|f(y)| d y \frac{r^{n+1}}{t^{1+(n / 2)}} e^{-c_{2} r^{2} / t} d r . \\
& \leq C \sup _{r \geq \sqrt{t}} \frac{1}{|B(0, r)|} \int_{B(0, r)}|f(y)| d y .
\end{aligned}
$$


Under the assumption on $f$ in the theorem, by the inequality just before (3.8),

$$
\frac{1}{|B(0, r)|} \int_{B(0, r)}|f(y)| d y \rightarrow 0, \quad r \rightarrow \infty .
$$

So $I(0, t) \rightarrow \infty$. Here we note that the just mentioned inequality is a direct result of the integration assumption on $K(x, r)$.

Therefore $u_{0}$ is a solution to (1.1). By the same argument from (3.9) to (3.12), we have

$$
\left|u_{0}(x)\right| \leq C(1+d(x))^{1+\alpha} \sup _{z} \int_{\mathbf{M}} \frac{d(z, y)}{d(z, y)^{\alpha}|B(z, d(z, y))|}|f(y)| d y \leq C(1+d(x))^{1+\alpha} .
$$

Step 4. We show that if $|f(x)| \leq \frac{C}{1+d(x)^{1-\epsilon}}$ with $\epsilon<\alpha$, then $f$ satisfies all the conditions of Theorem 1.2.

By the first inequality in (3.13), it is enough to prove that

$$
K \equiv \int_{\mathbf{M}} \frac{d(x, y)^{1-\alpha}}{|B(x, d(x, y))|} \frac{1}{1+d(y)^{1-\epsilon}} d y \leq C
$$

for all $x \in \mathbf{M}$. The proof, which is somewhat standard, is here for completeness.

Let us write

$$
K=\int_{d(y) \geq d(x, y) / 2} \ldots d y+\int_{d(y) \leq d(x, y) / 2} \ldots \equiv K_{1}+K_{2} .
$$

First we estimate $K_{1}$.

It is clear that

$$
\begin{aligned}
K_{1} \leq & \int_{\mathbf{M}} \frac{d(x, y)}{d(x, y)^{\alpha}|B(x, d(x, y))|} \frac{1}{1+d(x, y)^{1-\epsilon}} d y \\
=\Sigma_{k=1}^{\infty} & \int_{2^{k-1} \leq d(x, y) \leq 2^{k}} \frac{1+d(x, y)^{-(\alpha-\epsilon)}}{|B(x, d(x, y))|} d y \\
& \quad+\Sigma_{k=1}^{\infty} \int_{2^{-k} \leq d(x, y) \leq 2^{-k+1}} \frac{d(x, y)^{\alpha}}{|B(x, d(x, y))|} d y .
\end{aligned}
$$

By the doubling condition of geodesic balls, it is easy to see that

$$
K_{1} \leq C \Sigma_{k=1}^{\infty} \frac{1}{\left(1+2^{k-1}\right)^{\alpha-\epsilon}}+C \Sigma_{k=1}^{\infty}\left(1+2^{-k+1}\right)^{\alpha} \leq C,
$$

where $C$ is independent of $x$.

Next we estimate $K_{2}$.

When $d(y) \leq d(x, y) / 2$, we have, by Proposition 2.2 ,

$$
|B(x, d(x, y))| \sim|B(y, d(x, y))| \geq C \frac{d(x, y)}{d(y)}|B(y, d(y))| \sim C \frac{d(x, y)}{d(y)}|B(0, d(y))| .
$$

Hence, when $d(y) \leq d(x, y) / 2$, there holds,

$$
\frac{d(x, y)^{1-\alpha}}{|B(x, d(x, y))|} \leq \frac{d(y)^{1-\alpha}}{|B(0, d(y))|}
$$


This shows

$$
K_{2} \leq \int_{\mathbf{M}} \frac{d(y)^{1-\alpha}}{|B(0, d(y))|} \frac{1}{1+d(y)^{1-\epsilon}} d y \leq C,
$$

as proven in the last paragraph. By now, we have proven (3.14).

This proves part (a) and (b).

Proof of Theorem 1.2, part (c). The proof follows the same lines as part (a). let $u_{0}$ be the solution of (1.1), obtained in part (a). Note that $u_{0}$ is given by

$$
u_{0}(x)=\int_{\mathbf{M}} \int_{0}^{\infty}(G(0, s ; y, 0)-G(x, s ; y, 0)) d s f(y) d y
$$

Hence

$$
\left|\nabla u_{0}(x)\right| \leq \int_{\mathbf{M}} \int_{0}^{\infty}\left|\nabla_{x} G(x, s ; y, 0)\right| d s|f(y)| d y .
$$

Note that this is identical to the integral on the right hand side of (3.3), except the $x$ here is replaced by $z$. So following the proof of part (a) verbatim, we obtain

$$
\left|\nabla u_{0}(x)\right| \leq C\left(1+d(x)^{\alpha}\right) .
$$

This proves (c).

Finally we prove (d). This second derivative estimates are derived from some well known arguments (see [NST] and the reference there e.g.). For completeness we give a sketch here.

Since $\Delta u=f$, using local orthonormal frame, on has

$$
\frac{1}{2} \Delta|\nabla u|^{2}=\Sigma_{k, l} u_{k l}^{2}+\Sigma_{k} u_{k}(\Delta u)_{k}+\Sigma_{k, l} R_{k l} u_{k} u_{l} \geq\left|\nabla^{2} u\right|^{2}+<\nabla u, \nabla f>.
$$

Here $R_{k l}$ is the Ricci curvature tensor.

Let $\phi \geq 0$ be a smooth function with compact support in $B(0,2 R)$. Multiplying the above inequality by $\phi^{2}$ and integrating by parts, we get

$$
\begin{aligned}
\int_{B(0,2 R)} & \phi^{2}\left|\nabla^{2} u\right|^{2} \\
& \leq \int_{B(0,2 R)} \phi^{2} f^{2}+\int_{B(0,2 R)} \phi|\nabla \phi||\nabla u||f|+2 \int_{B(0,2 R)} \phi|\nabla \phi|\left|\nabla\left(|\nabla u|^{2}\right)\right| \\
& \leq \int_{B(0,2 R)} \phi^{2} f^{2}+\left(1+\epsilon^{-1}\right) \int_{B(0,2 R)}|\nabla \phi|^{2}|\nabla u|^{2}+\epsilon \int_{B(0,2 R)} \phi^{2}\left|\left(|\nabla u|^{2}\right)\right| .
\end{aligned}
$$

It follows that

$$
\int_{B(0,2 R)} \phi^{2}\left|\nabla^{2} u\right|^{2} \leq C \int_{B(0,2 R)} \phi^{2} f^{2}+C R^{-2} \int_{B(0,2 R)}|\nabla u|^{2} .
$$

Since, by (c), $|\nabla u(x)| \leq C(1+d(x))^{\alpha}$ with $\alpha<1$, we have

$$
\lim _{R \rightarrow \infty}|B(0, R)|^{-1} \int_{B(0, R)}\left|\nabla^{2} u\right|^{2}=0 .
$$


This finish the proof Theorem 1.2.

Now we are ready to give

Proof of Theorem 1.2'. This follows from Theorem 1.2 and [NST]. By Theorem 1.2 one can find a solution $u$ of $\frac{1}{2} \Delta u=f$. Here $f$ is the trace of $\rho$. Moreover $u$ satisfies (3.18). It is known that the function $\|\sqrt{-1} \partial \bar{\partial} u-\rho\|$ is subharmonic ([MSY] p187 e.g.). Fixing $x \in \mathbf{M}$ and choosing $R \geq 8 d(x)$, by the mean value property in [LS], one has

$$
\begin{aligned}
\| \sqrt{-1} \partial \bar{\partial} u & -\rho\left\|^{2}(x) \leq \frac{C}{|B(x, R)|} \int_{B(x, R)}\right\| \sqrt{-1} \partial \bar{\partial} u-\rho \|^{2} \\
& \leq \frac{C}{|B(x, R)|} \int_{B(x, R)}\left(\left|\nabla^{2} u\right|^{2}+\|\rho\|^{2}\right) \\
& \leq \frac{C}{|B(0,2 R)|} \int_{B(0,2 R)}\left(|f|^{2}+\|\rho\|^{2}\right)+\frac{C R^{-2}}{|B(0,2 R)|} \int_{B(0,2 R)}|\nabla u|^{2} .
\end{aligned}
$$

Letting $R \rightarrow \infty$, we find that $\sqrt{-1} \partial \bar{\partial} u-\rho=0$.

Proof of Theorem 1.3. (a). By Theorem 1.2' and the assumption on the Ricci curvature, there exists a strictly plurisubharmonic function as we can solve the PoincaréLelong equation by taking the Ricci tensor as the $\rho$. By [GW1], we can use the Busemann function to define a continuous plurisubharmonic exhaustion function. Now, by standard arguments in several complex variables, $\mathbf{M}$ is Stein.

(b). Let $p$ be the pole of $\mathbf{M}$. Then $\mathbf{M}$ is covered by all the rays $\gamma$ starting from $p$. Using this fact one can easily conclude (see [GW2]) the distance function $d(p, x)=$ $\sup _{\gamma}\left\{g_{\gamma}(x)\right\}$ is a continuous plurisubharmoic function, where $g_{\gamma}$ is the Busemann function defined with respect to $\gamma$. But $d(p, x)$ is an exhaustion function as $\mathbf{M}$ is complete. By Theorem 1.2', $\mathbf{M}$ admits a strictly plurisubharmoic function as in (a). By standard arguments in several complex variables, one concludes $\mathbf{M}$ is Stein.

4. Euclidean and flat cases. In this section, we establish the solvability of (1.1) when $f$ actually blows up near infinity. The result is obtained by extending Riesz's idea. Instead of using the formula

$$
u(x)=\int_{\mathbf{M}}[G(0, y)-G(x, y)] f(y) d y
$$

we look for $u$ in the form of

$$
u(x)=\int_{\mathbf{M}} T(x, y) f(y) d y
$$

where $T(x, y)$ is a Taylor expansion of $G(x, y)$ around 0 . This method allows (1.1) to be solved for any $f$ with polynomial growth near infinity. However it requires higher order estimates on $G(x, y)$ and the existence of special structures such as certain global harmonic coordinates. Even though the result is a little limited as far as the types of manifolds involved, it indicates an effective method of solving (1.1) for a much wider class of $f$, under certain structural condition of the manifolds. We expect many applications of the technique for general equations and systems that involve the Poisson equation. Let us note that for compact manifolds, there is a necessary and 
sufficient condition for the solvability of $\Delta u=f$. See [Au]. We need to mention that in the Euclidean case, this equation is solvable for all $f \in L_{l o c}^{1}$. This was proven, for example in $[\mathrm{Wx}]$.

THEOREM 4.1. Let $\mathbf{M}$ be a complete noncompact, non-parabolic manifold with nonnegative Ricci curvature. Suppose (i) $\mathbf{M}$ has a global harmonic coordinate, meaning the coordinate functions are harmonic; (ii) components of derivatives (of any order) of harmonic functions are harmonic. Then Given any $f \in C_{\text {loc }}^{\alpha}\left(\mathbf{M}^{n}\right)$ such that $|f(x)| \leq C\left(1+d(x)^{m}\right)$ for $m>0$, equation (1.1) has a solution $u \in C_{\text {loc }}^{2, \alpha}(\mathbf{M})$ satisfying $|u(x)| \leq C(1+d(x))^{m+2+\delta}$.

Proof. For simplicity we will only prove the Euclidean case. The general case is identical.

For $R>0$, let $G_{R}$ be the Green's function in $B(0, R)$ with zero boundary value and let

$$
\begin{aligned}
u_{R}(x)=-\int_{B(0, R)}\left\{G_{R}(x, y)-\left[G_{R}(0, y)+\nabla G_{R}(0, y) x+\ldots\right.\right. \\
\left.\left.\quad+\frac{x_{i_{1} \ldots x_{i_{k}}}}{k !} D_{x_{i_{1}} \ldots x_{i_{k}}}^{k} G_{R}(0, y)\right]\right\} f(y) d y \\
\equiv-\int_{B(0, R)} T(x, y) f(y) d y .
\end{aligned}
$$

Here and later, all derivatives are on the first entries of $G_{R}$.

Since one can always solve the Poisson equation with compact data, we assume, without loss of generality, that $f(x)=0$ in the ball $B(0,1)$.

We claim that (i) $\left|u_{R}(x)\right| \leq C(|x|)$ and that (ii) $\Delta u_{R}(x)=f(x)$ for $x \in B(0, R)$. Once the claim is proven, by standard compactness arguments, there exists a subsequence $u_{R_{k}}$ that converges pointwise to a function $u$ in $\mathbf{R}^{n}$. Clearly $\Delta u=f$.

First let us prove claim (i). From (4.1), we have, as before

$$
\begin{aligned}
u_{R}(x) & \leq \int_{B(0, R)-B(0,2 r)}\left|T(x, y)\left\|f(y)\left|d y+\int_{B(0,2 r)}\right| T(x, y)\right\| f(y)\right| d y \\
& \equiv I_{1}+I_{2} .
\end{aligned}
$$

Here $r=|x|$ and $R>>r$.

We estimate $I_{1}$ first. Let us recall the following well-known facts: for $y \in B(0, R)-$ $B(0,2 r)$ and $z \in B(0, r)$,

$$
\left|D_{x_{i_{1} \ldots x_{k}}}^{k} G_{R}(z, y)\right| \leq \frac{C_{k, n}}{|x-y|^{n-2+k}} .
$$

This can be proven by using either the explicit formula for $G_{R}$ or using integration by parts and Moser's iteration. Here the constants are independent of $k$.

Using Taylor expansion, there is $\xi \in B(0,|x|)$ such that

$$
|T(x, y)|=\frac{1}{(k+1) !}\left|\xi_{i_{1}} \ldots \xi_{i_{k+1}} D_{\xi_{i_{1}} \ldots \xi_{i_{k+1}}}^{k} G_{R}(\xi, y)\right| \leq \frac{C n^{k+1}|x|^{k+1}}{(k+1) !|y|^{n-1+k}} .
$$

Here we have used the inequality $|y-\xi| \geq|y| / 2$. It follows that

$$
I_{1} \leq \frac{C n^{k+1}|x|^{k+1}}{(k+1) !} \int_{B(0, R)-B(0,2 r)} \frac{f(y)}{|y|^{n-1+k}} d y .
$$


By the bound $|f(y)| \leq C\left(1+|y|^{m}\right)$, we have, by choosing $k=m+1+\delta$ with $\delta>0$,

$$
I_{1} \leq \frac{C n^{k+1}|x|^{m+2+\delta}}{(k+1) !} .
$$

For $I_{2}$, we proceed as follows

$$
I_{2} \leq \int_{B(0,2 r)-B(0,1)} \sum_{j=0}^{k} \frac{C_{j}}{|y|^{n-2+j}}|x|^{j}|f(y)| d y .
$$

Hence

$$
I_{1} \leq \frac{C n^{k+1}|x|^{m+2+\delta}}{(k+1) !}
$$

Combining (4.3) and (4.4), we have proven claim (i).

Next we prove claim (ii), where we will use an interesting cancellation property. Clearly

$$
\Delta u_{R}(x)=f(x)-\int_{B(0, R)-B(0,1)}\left[\Delta J_{1}+\ldots+\Delta J_{k}\right] f(y) d y
$$

where

$$
J_{k} \equiv \frac{x_{i_{1} \ldots x_{i_{k}}}}{k !} D_{x_{i_{1} \ldots x_{i_{k}}}}^{k} G_{R}(0, y)
$$

Obviously $\Delta J_{1}=0$ since $\Delta x_{i}=0$. Next

$$
\Delta J_{2}=\Delta\left(x_{i_{1}} x_{i_{2}} D_{x_{i_{1}} x_{i_{2}}}^{2} G_{R}(0, y)\right) d y=2 \delta_{i_{1} i_{2}} D_{x_{i_{1}} x_{i_{2}}}^{2} G_{R}(0, y)=2 \Delta G_{R}(0, y)=0
$$

By direct computation, we see that

$$
\Delta J_{3}=\Delta\left(x_{i_{1}} x_{i_{2}} x_{i_{3}} D_{x_{i_{1}} x_{i_{2}} x_{i_{3}}}^{2} G_{R}(0, y)\right)=6 \Sigma_{i=1}^{n} x_{i} D_{x_{i}} \Delta G_{R}(0, y) .
$$

Since $\Delta_{z} G_{R}(z, y)=0$ when $z \neq y$, we have $\Delta J_{3}=0$.

By induction

$$
\begin{aligned}
\Delta J_{k} & =\Delta\left(\frac{x_{i_{1} \ldots x_{i_{k}}}}{k !}\right) D_{x_{i_{1}} \ldots x_{i_{k}}}^{k} G_{R}(0, y) \\
& =\frac{k(k-1)}{k !} \Sigma x_{i_{1} \ldots x_{i_{k-2}}} D_{x_{i_{1}} \ldots x_{i_{k-2}}}^{k-2} \Delta G_{R}(0, y) f(y) .
\end{aligned}
$$

Since $\Delta_{z} G_{R}(z, y)=0$ when $z \in B(0,|x|)$ and $y \geq 2|x|$, we have $\Delta J_{k}=0$. This proves $\Delta u_{R}=f$.

Acknowledgement We thank Professors Peter Li, Lei Ni and $\mathrm{H}$. Wu for helpful conversations. Special thanks goes to Professor S. T. Yau for his support on the paper.

\section{REFERENCES}

[Au] Aubin, THIERry, Some nonlinear problems in Riemannian geometry, Springer Monographs in Mathematics. Springer-Verlag, Berlin, 1998.

[C] Calabi, Eugenio, On Ricci curvature and geodesics, Duke Math. J., 34 (1967), pp. 667-676. 
[CY] Cheng, S. Y.; YAU, S. T., Differential equations on Riemannian manifolds and their geometric applications, Comm. Pure Appl. Math., 28:3 (1975), pp. 333-354.

[CZ] CHEn, B. L. AND X. P. ZHI, On complte Kähler manifolds with positive bisectional curvature, preprint.

[GW1] Greene, R. E.; Wu, H., $C^{\infty}$ convex functions and manifolds of positive curvature, Acta Math., 137:3-4 (1976), pp. 209-245.

[GW2] GREENE, R. E.; WU, H., On Kähler manifolds of positive bisectional curvature and a theorem of Hartogs, Special issue dedicated to the seventieth birthday of Erich Khäler, Abh. Math. Sem. Univ. Hamburg, 47 (1978), pp. 171-185.

[LS] LI, PETER; SCHOEN, RICHARD, $L^{p}$ and mean value properties of subharmonic functions on Riemannian manifolds, Acta Math., 153:3-4 (1984), pp. 279-301.

[LY] P. LI AND S. T. YAU, On the parabolic kernel of the Schrödinger operator, Acta Math, 156 (1986), pp. 153-201.

[M] MoK, NGAIming, An embedding theorem of complete Kähler manifolds of positive bisectional curvature onto affine algebraic varieties, Bull. Soc. Math. France, 112:2 (1984), pp. 197-250.

[MSY] N. MoK, Y. T. SiU AND S. T. YAU, The Poincaré-Lelong equation on complete Kähler manifolds, Compositio Math., 44 (1981), pp. 183-218.

[Ni] L. NI, Poisson equation and Hermitian-Einstein metrics on Holomorphic vector bundles over complete noncompact Kähler manifolds, preprint 2001.

[NST] L. NI, Y. Shi AND L. F. TAM, Poisson equation, Poincaré-Lelong equation and curvature decay on complete Kähler manifolds, J. Diff. Geom., 52:2 (2001), pp. 339-388.

[S] Shen, Zhongmin, Complete manifolds with nonnegative Ricci curvature and large volume growth, Invent. Math., 125:3 (1996), pp. 393-404.

[Sh] SHI, WAN-XIONG, Ricci flow and the uniformization on complete noncompact Kh/"aler manifolds, J. Differential Geom., 45:1 (1997), pp. 94-220.

[SY] Schoen, R.; YAu, S.-T., Lectures on Differential Geometry, International Press, Cambridge, MA, 1994.

[Wa] WANG, JIAPING, The spectrum of the Laplacian on a manifold of nonnegative Ricci curvature, Math. Res. Lett., 4:4 (1997), pp. 473-479.

[Wx] WANG, X. J., personal communication.

[Wu1] Wu, H., An elementary method in the study of nonnegative curvature, Acta Math., 142:1-2 (1979), pp. 57-78.

[Wu2] Wu, H., On certain Khäler manifolds which are q-complete, Complex analysis of several variables (Madison, Wis., 1982), pp. 253-276, Proc. Sympos. Pure Math., 41, Amer. Math. Soc., Providence, RI, 1984.

[Zh] QI S. ZHANG, Large time behavior of Schrödinger heat kernels and applications, Comm. Math. Phy., 210 (2000), pp. 371-398. 